\title{
Science Abstract Model Simulation Framework
}

\author{
Amir M. Abdol ${ }^{1}$, Jelte M. Wicherts ${ }^{*^{*}}$ ॥
}

1 Tilburg School of Social and Behavioral Sciences, Tilburg University, Tilburg, The Netherlands

1i@amirmasoudabdol.name

*j.m.wicherts@tilburguniversity.edu

\section{Abstract}

Meta-researchers increasingly study biases in quantitative study outcomes (effect sizes) that emerge from questionable research practices (QRPS) in designing, running, analyzing, and reporting studies. Here, we introduce an extensible and modular $\mathrm{C}++$ simulation framework called SAM (Science Abstract Model) that enables systematic study of the effects of QRPs and researchers' degrees of freedom ( $p$-hacking) on a host of outcomes across the different phases of quantitative studies that test hypotheses. SAM achieves this by modular modeling of different entities and processes involved in research, from study designs and inferential criteria, the data collection and analyses, to the submission and acceptance of manuscripts in a journal. We demonstrate the advantages of our approach by reproducing and extending the Bakker, van Dijk, and Wicherts (2012) simulation study that investigated the effects of various $p$-hacking methods and publication bias on meta-analytic outcomes. We showcase how SAM's modularity and flexibility makes it possible to easily examine the original study by modifying, adding, or removing different components- e.g., publication bias, different significance levels, or meta-analytic metrics. We focus our illustration on the fundamental question of whether lowering $\alpha$ will reduce the biases in the scientific literature. 


\section{Introduction}

Meta-researchers aim to study the processes of contemporary research to better understand biases and inefficiencies emerging from how studies are designed, data are analyzed, results are reported, and outcomes are disseminated, and to develop methods, tools, and practices that heighten the efficiency, trustworthiness, and eventual impact of research [1]. In quantitative hypothesis-testing research, the most well-known and widely studied biases include the common use of underpowered and poorly controlled study designs, publication bias of studies based on significance, the selective reporting of desirable outcomes within studies, explorations of data incorrectly presented as not being mainly data-driven, improper use of inferential statistics, and biases emerging from the opportunistic use of flexibility of the analyses of quantitative data in order to achieve thresholds for statistical significance ( $p$-hacking) [2, 3]. Meta-researchers often employ formal statistical approaches to better understand how such biases emerge, what their effects are on validity (or replicability) of single studies and on sets of studies as they reported in the literature and/or included in meta-analyses. Subsequently, they develop improved methodological practices, research tools, and statistical approaches to help avoid, detect, and correct the emerging biases. For instance, early studies in the field highlighted the effects of selective reporting of studies that yielded significant results over non-significant results, developed tests to detect such publication bias in the context of meta-analyses, and developed statistical corrections for the emerging overestimation of effect sizes because of publication bias [4]. More recent meta-research focused on biases emerging from common yet substandard methods to analyze data and reported specific outcomes within these studies. These studies have yielded important insights on the oftentimes large biases emerging from publication bias and $p$-hacking and widespread calls to avoid them by open prior registration of studies and analytic protocols [5-7].

Although formal statistical approaches often allow one to derive specific implications of particular (often singular) biases emerging from substandard research practices, the statistical tool of Monte Carlo simulation studies has become the main workhorse of meta-researchers to understand such biases and to find solutions [8]. Simulations allow researchers to specify the true population values of main parameters of interest, to see whether a set of studies subject to biasing factors can be uncovered accurately and precisely. For instance, a simulation study uses the genuine effects of an intervention (or actual distribution of such effects in case they vary in the 
population) and subsequently determines how a particular level of publication bias (e.g., a scenario wherein $50 \%$ of the non-significant outcomes are included) inflates effect size estimates in a fixed effect (or random effect) meta-analysis. Such simulations enable more complex scenarios in which multiple sources of bias are included to study their relative effects and interactions. The ability of these simulations to realistically test what happens in actual research practice rests on the realism of their design.

Not all aspects of previous simulation studies are indeed realistic. For instance, while researchers are continuously developing new tools, tests and methods to detect and account for biases affecting our research and induced in publications [9-13], we are far from drawing the full picture and ultimately coming to agreements on possible solutions or counter measures. Some researchers are advocating for the reduction of alpha in significance testing [14], while others [15] even called for the complete abandonment of significance testing, or argued in favor of Bayesian approaches [16-18]. Lastly, a more pragmatic group of researchers are working toward promoting new approaches and tools such as pre-registration [19], registered reports [20], multiverse and multiverse analysis [21] and many labs replications [22].

Despite our differences, disagreements and skepticisms [23, 24], meta-scientists are ultimately working toward identifying and accounting for various forms of biases affecting scientific findings. In the case of the publication bias where its severity and seemingly endless train of positive and significant outcomes raised every existing flag [25-27], we now acknowledge the problem and collectively working toward solutions [1]. However, not all biases are introduced by or perhaps accumulated at publications and journals; as researchers' degrees of freedom extend throughout the entire process of conducting research, they tend to sprinkle their effects and biases over a study and its outcome along the way [2]. Studying and exposing these biases throughout this vast universe of methods, practices, and decisions, a.k.a., doing research, is undeniably not a straightforward task especially considering the scarcity of the data.

Meta-scientists, if fortunate, will only have the raw data to work with, and often there is little to no data available on the processes and decisions that an individual researcher utilizes throughout their research [28]. This lack of data makes it challenging to identify, correct or even agree on the effect of questionable research practices; in fact, this has reportedly even led to researchers defending their use cases [29]. In order to address this issue, meta-scientists are activity advocating for methods like pre-registration and registered report [30, 31] in order to be able to observe researchers' choices and decisions during their research and hopefully improve 
the quality and quantity of our data.

In the meantime, in order to fill the gap in the data and study the understudied corners in this complex system, we are increasingly relying on simulation studies to model, experiment and examine the effects of researchers' degrees of freedom. For example, a simulation study by Bakker et al. [32] concludes that its favorable for a researcher to perform a number of smaller studies with low statistical power, instead of one study with a high power, if the goal is to find significant results and get published; or, a simulation study by Friese et al. [11] demonstrate how bias introduced by $p$-hacking methods will find its way into meta-analytic effect size estimates.

However, the majority of these simulation studies are relatively limited, often unrealistic, and without exception model everything as a strictly linear process which indicates that they fail to fully mimic and capture researchers intricate decision-making processes. In addition, often for performance reasons or to shun complexity, many studies do not choose to fully model certain processes. This is especially common in modeling $p$-hacking, where the effect of most $p$-hacking methods is mimic by simply pushing the $p$-value below the significance level [11]. This practical yet entirely unrealistic approach toward modelling $p$-hacking assumes that most researchers' degrees of freedoms are merely a form of rounding; and therefore, fail to capture the full effect of $p$-hacking methods on various aspects of a research as a process. In addition, the lack of common standards and simulation framework make their results inconsistent, open to criticism, and untransferable. For instance, it is difficult to know whether or not the type of QRPs that Bakker et al. have applied in their research will have the same propagative effects on meta-analytic estimators studied by Friese et al. [11].

In this paper, we directly address the aforementioned issues by introducing SAM, a simulation framework that is designed to simulate the entire process of conducting scientific research without compromising on complexity. SAM is a modular, flexible and extensible simulation framework that exposes many components and processes of the study; and allows the user to experiment, tweak and examine their behaviors individually and collectively. In order to be able to simulate the research process, SAM models a wide range of methods and procedures involved in research. In the Materials and Methods section, we elaborate on these components and show how this modular approach helps us design and simulate the entire process of conducting research, from the moment that a researcher formulates his/her study design, to the point where his/her research is being evaluated by the Journal. Furthermore, we demonstrate how the flexibility of each component allows us to fine-tune different scenarios (e.g., modeling a specific 
outlier removal approach) and isolate certain behaviors. In the Results section, we use SAM to first reproduce and then extend a previous simulation study [32] by delving deeper into the original design and showcasing how the versatility of SAM may help us answer new questions such as whether or not lowering the $\alpha$-level reduces the biases reported in their study, and how the whole system behaves in the presence of the publication bias.

\section{Materials and Methods}

In order to understand the basis of biasing effects of researchers' questionable research practices and journal's reviewing policies, we need to model the entire process of conducting research. In this section, we describe how components and procedures of this process are conceptualized in SAM. We discuss how the concept of modularity allows us to customize different parts of the system, and how the notion of separation of concerns helps us to achieve a realistic and familiar simulation by delegating different parts of the process to its relevant actors, e.g., Researchers and Journals.

In this article, we may use the "the process" to refer to "the process of conducting research". We use Title Case styling whenever we are referring to one of SAM's modules, e.g., Review Strategy. In addition, we use the female pronoun when we are describing the processes from Researcher's perspective. Furthermore, for the purpose of consistency, we will be using the term "Strategy" as a universal term for methods, models, or algorithms, e.g., $T$-test as a Test Strategy or Cohen's d as an Effect Strategy. Furthermore Finally, it is worth noting that SAM's modules are abstract representations of different components of the process, and they may not exactly correspond to their real-world counterparts.

\section{The Overview}

A typical study within the dominant hypothetical-deductive tradition starts by formulating a hypothesis when a Researcher jots down her ideas about a certain empirical (i.e., testable) phenomenon. In order to test this hypothesis, she sets up an experiment in which the underlying parameters and boundaries of her theories and ideas are defined, i.e., Experiment Setup. Next, based on the specification of her design, the actual Experiment will be conducted where the Researcher - in accordance with the Experiment Setup - collects certain types of data, e.g., sampling data, questionnaire results, sensory data, or images, in order to test her hypothesis. The 
next step is the (pre-)processing and analysis of the data that eventually leads to conclusions regarding the initial hypothesis, i.e., research findings. If these conclusions or results are informative -regardless of their agreement or disagreement with the initial hypothesis, i.e., pre-registered outcome- the Researcher selects a Journal and sends her findings (in the form of a Manuscript) to be reviewed according to Journal's Review Strategy. At last, the Journal will decide on whether the submitted Manuscript is going to be published and be considered a Publication.

Fig 1 shows the schematic overview of this process. Notice that we have broken the process into three main stages, preparing, performing, and publishing the research. The Researcher oversees the first two stages, while the last stage is performed by the Journal. The Researcher and the Journal are the only players, i.e., agents, involved in this process. They are the ones controlling and interacting with other components. They can modify other components, pass them to each other, and make certain decisions based on their contents. For instance, the Researcher may decide on whether she wants to apply certain $p$-hacking methods on her Experiment, or the Journal may be equipped with a specific Review Strategy to evaluate the submitted Manuscript based on different criteria.

Fig 1. Overview of the Research Process. A high-level representation of research components, processes and players. While the Researcher is responsible for preparing and performing the research, the Journal is taking care of the reviewing and publishing tasks.

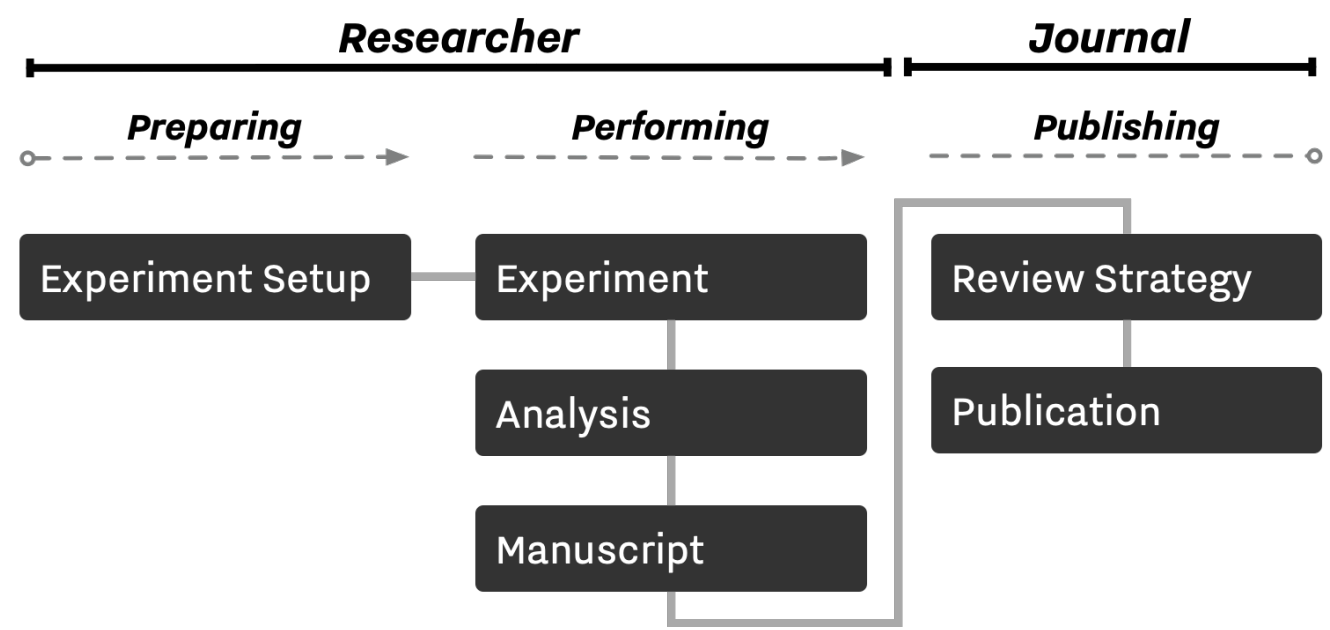

\section{The Modules}

SAM consists of 3 primary modules or components: Experiment, Researcher and Journal. Each 
as shown in Fig 1. Every primary component of SAM consists of several smaller subcomponents,

and those may be made of smaller parts themselves, as shown and color-coded in Fig2. Here, we introduce these modules by outlining their definition, role and design criteria. In addition, we provide a catalog of these modules as well as a list of available methods and their corresponding parameters in Table 1. Note that the user of SAM is the one in charge of all these components and processes, it is the user that configures every module based on his/her preferences and design of the simulation study.

Fig 2. Overview of SAM's Primary and Secondary Components. While the Researcher and Journal are responsible for running the simulation scenario, the Simulation Module controls the total number of simulations and makes sure that the correct outputs are generated and exported at the end of the simulation. The depicted indentation represents the hierarchy between the modules.

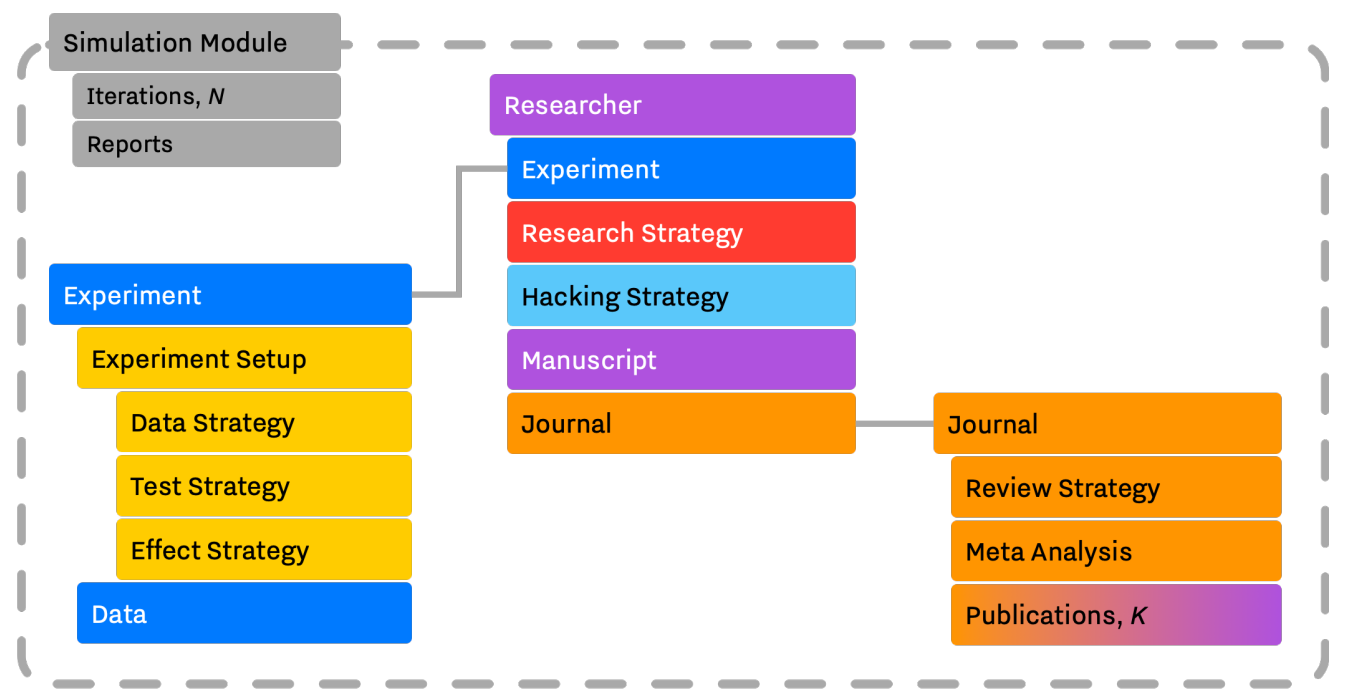

The Experiment module comprises of several sub-modules, each dealing with different aspects of performing an experiment. It contains a reference to the Experiment Setup, i.e., it knows the design. This allows the Researcher to access the specification of the study design at any time.

- The Experiment Setup holds the specification of the study design, e.g., number of groups, number of dependent variables, number of items. In addition, it is aware of the study population (i.e., the true effect), the selected test method, and other constructs that the Researcher may have in mind/place to define and constrain her research. In fact, the Experiment Setup design is closely related to the concept of pre-registration, and it can be utilized by the user to model the concept of pre-registration, if needed. 
Table 1. Selected SAM's primary and secondary modules and their available methods, and their parameters. For the up-to-date list of modules and methods, please visit our website.

\begin{tabular}{|c|c|c|c|}
\hline Components & Subcomponents & Available Methods & Description and Parameters \\
\hline \multirow[t]{5}{*}{ Experiment Setup } & Design Parameters & $\begin{array}{l}\text { \# Groups, \# DVs, \# Reps, \# Items, } \\
\text { \# Observations }\end{array}$ & Number of observations per DV can extensively be customized, or randomized. \\
\hline & \multirow[t]{2}{*}{ Data Strategy } & Linear Model & $\begin{array}{l}\text { Generates data based on linear model, } \bar{y}=\bar{x}+\bar{\epsilon} \text {, using a multivariate normal } \\
\text { distribution } \bar{x} \sim \mathcal{N}(\mu, \Sigma), \bar{\epsilon} \sim \mathcal{N}\left(\mu_{\epsilon}, \Sigma_{\epsilon}\right) \text {. Alternatively, a set of } \\
\text { univariate distributions can be defined and assigned to individual dependent } \\
\text { variables. }\end{array}$ \\
\hline & & Graded Response Model & $\begin{array}{l}\text { Generates data based on GGRM 33]. Rasch Model is used as the default } \\
\text { score function [34. }\end{array}$ \\
\hline & Test Strategy & \multicolumn{2}{|c|}{$T$-Test, $F$-Test, Yuen $T$-Test, Wilcoxon Test } \\
\hline & Effect Strategy & \multicolumn{2}{|c|}{ Mean Differences, Std. Mean Differences, Cohen's d, Hedges' g } \\
\hline Data & \multicolumn{3}{|c|}{ Stores the raw data, descriptive statistics, test statistics, and meta-data necessary to the Researcher. } \\
\hline Research Strategy & Selection-Decision & $\begin{array}{l}\text { Initial } \\
\text { Before } p \text {-Hacking } \\
\text { While } p \text {-Hacking } \\
\text { After } p \text {-Hacking } \\
\text { After Replication } \\
\text { Before Submission }\end{array}$ & $\begin{array}{l}\text { A set of logical rules defining a series of Selection-Decision Strategies at } \\
\text { different stages of the Research Strategy. The Selection is a set of logical } \\
\text { expression that is being used to filter a certain outcome, e.g., [ "pvalue }< \\
0.05 ", \text { "effect }<0.2 ", \text { " min (random) "]. A Decision is a set of logical } \\
\text { expression used for evaluating the previously selected outcome, e.g., } \\
\text { ["pvalue }<0.05 ", \text { "effect }<0.2 "] \text {. See Fig. } 1\end{array}$ \\
\hline \multirow[t]{11}{*}{ Hacking Strategies } & \multirow[t]{8}{*}{ Strategies } & Selective Reporting* & $\begin{array}{l}\text { Selective reporting can be achieved by customizing selection-decision se- } \\
\text { quences and designing a specific Research Strategy. }\end{array}$ \\
\hline & & Optional Stopping & $\begin{array}{l}\text { Adds new observations to each DV. Several aspects of the algorithm can be } \\
\text { customized, e.g, the number of new observation, the number of attempts, } \\
\text { the stopping condition, and the target DVs }\end{array}$ \\
\hline & & Subjective Optional Stopping & $\begin{array}{l}\text { Specialized version of the above method where the stopping_condition is } \\
\text { set to ["sig"]. }\end{array}$ \\
\hline & & Outliers Removal & $\begin{array}{l}\text { Removes the outliers from the data. Several aspects of the algorithm can be } \\
\text { customized, e.g., the number of obs. to be removed, distance from the mean, } \\
\text { the number of attempts, target and the stopping condition. }\end{array}$ \\
\hline & & Subjective Outliers Removal & $\begin{array}{l}\text { Specialized version of the above method where multiple K's can be set, and } \\
\text { stopping_condition is used to stop the algorithm as soon as certain criteria } \\
\text { is met. }\end{array}$ \\
\hline & & Optional Dropping & $\begin{array}{l}\text { Filters the data based on the given covariate, and replaces the Experiment } \\
\text { with the result. Several customizable parameters are available. }\end{array}$ \\
\hline & & Group Pooling & $\begin{array}{l}\text { Combines dependent variables of two more groups, and adds the newly } \\
\text { generated group to the Experiment. }\end{array}$ \\
\hline & & Questionable Rounding & $\begin{array}{l}\text { Rounds the } p \text {-value based on the given criteria. Customizable parameters } \\
\text { include: target } p \text {-value, trigger (distance from the } p \text {-value to initiate the } \\
\text { procedure), method of rounding, e.g., soft, hard. }\end{array}$ \\
\hline & \multirow[t]{3}{*}{ Behavioral Parameters } & Prob. of Performing a Strategy & $\begin{array}{l}\text { A parameter indicating whether the Researcher is going to enter the QRP } \\
\text { procedure. In addition to True or False, this value can be randomized for each } \\
\text { Researcher. }\end{array}$ \\
\hline & & Prob. of Committing to a Strategy & $\begin{array}{l}\text { A parameter indicating whether the Researcher decides to apply the current } \\
\text { QRP to the Experiment. This can be used to adapt Researcher's decision } \\
\text { to the content of the Experiment, e.g., only if effect is further than a certain } \\
\text { distance from the target effect. }\end{array}$ \\
\hline & & $\begin{array}{l}\text { Order and Execution technique of } \\
\text { strategies }\end{array}$ & $\begin{array}{l}\text { A set of parameters indicating the order of applying a set of strategies on an } \\
\text { Experiment. In addition, the Researcher can be configured to either apply the } \\
\text { strategies on top of each other, or one by one on a new copy of the Experiment. }\end{array}$ \\
\hline \multirow[t]{4}{*}{ Review Strategy } & \multirow[t]{4}{*}{ Strategies } & Free Selection & No filtering is imposed by the Journal. \\
\hline & & Based on an user-defined logic & Manuscripts may be accepted if certain logical expression is satisfied. \\
\hline & & Significant Based Review Strategy & $\begin{array}{l}\text { Manuscripts may be accepted if they are significant, if not, they have } \mathrm{P}_{\mathrm{b}} \\
\text { chance of being accepted. }\end{array}$ \\
\hline & & Random Acceptance Rate & Manuscripts may be accepted based on a draw from a $U(0,1)$ \\
\hline Meta-Analysis & Methods & $\begin{array}{l}\text { Random-Effect, Egger's Test, Cor- } \\
\text { relation Test, Trim and Fill }\end{array}$ & $\begin{array}{l}\text { At the end of each simulation cycle, the Journal calculates the selected } \\
\text { estimate and saves it for later analysis. }\end{array}$ \\
\hline Pubs. Pool Size & & e J & g one simulation cycle. \\
\hline
\end{tabular}


- The Data Strategy contains the information about the population of the study. For instance, this information entails the true effect sizes and the actual measurement characteristics of the measures used in the study. It is the source of data that the Researcher uses to collect sample data for her Experiment. Every time that the Researcher asks for data-points, the Data Strategy generates a sample from the population and delivers it to the Researcher. (Note that the Data Strategy can be customized by the user to simulate various types of designs, analysis and data, including SEM approaches and mediation analysis).

- The Test Strategy is how the hypothesis is going to be evaluated, e.g., a $T$-Test to study the mean difference on an outcome variable between two experimental conditions.

- The Effect Strategy is the method of calculating the effect size, e.g., Cohen's D.

- The Data module is a container of the study data-points. The Researcher can populate it sample from it, or alter it by adding, removing and even modifying individual data-points, if she wants to. You may think of it as the Excel sheet that a researcher uses to store his/her data.

The Researcher module, as our first player, is designed to imitate the behavior of a researcher. The Researcher controls the Experiment module. It runs the experiment based on the specification of the Experiment Setup. It populates the Data object (by asking for a sample from the Data Strategy), and it calculates and stores the statistics, effect sizes, etc. into the Experiment module. Next, based on a series of customizable rules and criteria, it may decide to tinker with the Experiment -at different stages of its lifespan- in order to achieve a certain set of goals, e.g., $p$-value $<0.05$ \& effect $>0$.

- The Research Strategy is the underlying logics and rules that defines Researcher's thought process throughout her research. These rules allow the Researcher to observe and alter the Experiment based on her judgments and goals. Note that these rules can be customized by the user to model a variety of scenarios and thought processes, as we discuss in the coming section. For instance, the Researcher may decide to replicate the Experiment or choose between hacked outcomes if she finds it beneficial.

- The Hacking Strategy is a list of questionable research practices in the Researcher's 
arsenal. The Researcher, at various stages of conducting research, can reach out to these

methods and use them in order to alter the state of the Experiment. Note that the user can select and customize various methods, decide their order, their intensity and the stage where the Researcher should execute them.

- The Manuscript is the report that the Researcher prepares at the end of her research. In its simplest form it contains one (or more) outcome variable(s) that she is planning to submit to the Journal for review. The Manuscript is prepared by the Researcher based on her preferences, e.g., (effect $>0.3 \& p$-value $<0.05)$. Note that this query may result in multiple outcomes. This is an acceptable behavior in SAM and the user must account for it by equipping the Journal with a Review Strategy capable of evaluating multiple outcomes, if needed.

- The Researcher has a link to the Journal of her choice for when she is going to submit her Manuscript. The Researcher can communicate with the Journal by submitting her Manuscript and inquiring Journal's verdict.

The Journal module, as our second player in the process, is responsible for reviewing the incoming manuscripts and storing the accepted ones. Note that unlike a real scientific journal that may cover a wide range of research tracks, SAM's Journal module, in its current implementation, assumes that all submitted publications are from one research track. In other words, SAM's Journals are mainly acting as a pool for related studies ready to be analyzed using meta-analytic methods.

- The Review Strategy is internal logics and rules behind the Journal's reviewing policies. For instance, a Journal may be equipped with a Review Strategy that favors significant findings, i.e., imposing publication bias at certain rate, $\mathrm{P}_{\mathrm{b}}$. Note that like other strategies, these strategies and their rules can be fully customized by the user, and as we mentioned they can be designed to process and evaluate more than one outcome variable.

- The Publications is a list of accepted Manuscripts.

- The Meta-Analysis Strategy is a list of available meta-analytic metrics and/or publication bias estimators that the Journal uses to calculate, store, and report at the end of every simulation run, e.g., Egger's Test, Random-effect Estimate. 
The Simulation module, in contrast to other modules, is not involved in the research process. $\quad 219$ As depicted in Fig 2, it is the container of everything, and it controls high-level parameters and 220 specifications of the simulator program.

- The Iterations parameter indicates how many times each scenario should be repeated. For 222 instance, if the number of iterations is $\mathrm{N}$ and the Journal is collecting K publications in every $\quad 223$ run, in total $\mathrm{N} \times \mathrm{K}$ records will be reported in the final exported output file, e.g., CSV file. ${ }_{224}$

- The Report module can be used to indicate the types of data that the Journal is going to 225 export at the end of the simulation, e.g., meta-analysis data, list of publications (and their $\quad 226$ effect size, $p$-value, variance, number of observations, etc.), the history and details of every 227 Experiment submitted by the Researcher (whether they are hacked or not, actual data 228 points, general statistics, etc.).

\section{The User's Perspective}

As we mentioned in various points, the user of SAM can individually customize and alter the behaviors of every module and sub-module discussed in Fig 2, It is by these customization that users can create new Researcher(s) with different set of Hacking and Research Strategies, and behaviors in order to model certain simulation scenarios. For example, users may take advantage of the General Graded Response Model in order to design an IRT model based on the Rasch Model, or perhaps configure a specific covariance matrix to achieve a certain SEM using the Linear Model module. At the same time, users may choose a set of Hacking and Research Strategies in order to simulate a certain behavior. As we will clarify, the user is always in charge of the simulation design, and he/she has complete control over all and every module, method and processes.

\section{The Processes}

So far, we have introduced various modules, methods and players of SAM. In this section, we are going to delve deeper into the process of producing research by describing how all these pieces communicate with each other and eventually model a user-defined simulation scenario, Fig 3 . However, before exploring the simulation timeline and exposing different sub-processes of the research process, we need to introduce an important concept that gives the Researcher her reasoning ability, i.e., her brain. 


\section{The Selection-Decision Sequence, SDS}

The Researcher is the one who orchestrates the entirety of the research from the start until she prepares her Manuscript and passes it to the Journal. During this process, she ought to perform several decisions, at different stages. For instance, she needs to decide whether she is going to submit a certain outcome variable for the review or that she is not happy with the current quality of the outcome and decides to put it in the file-drawer. Or perhaps, she may choose to add a few new observations to the Experiment and increase her chances of finding a "better" outcome. In all these examples, the Researcher bases her decision on the properties and quality of available outcome variables or her goals. For example, she may only select the first dependent variable and base her decision on that, or she may first select all the dependent variables with positive effect size and then decide her next move (e.g., removing outliers) based on their $p$-values. This pattern of "making a decision after performing a selection" repeats itself in various stages of the research process; so much so that we decided to give this routine a name, i.e., Selection-Decision Sequence, and use it across the board as the main ingredient of Researcher's decision-making routine. As we describe the research workflow in the upcoming section, the Researcher uses a series of selection-decision sequences to navigate through her research workflow and choose between her options. Note that the user can customize every instance of selection and decision by constructing different queries using various variables (pvalue, effect, sig, hacked, etc.), operators (>, <, >=, etc.) and functions (min, max, random, etc.), e.g., ["effect > $\odot "$, "sig", "min (pvalue)"] for selecting the outcome with minimum $p$-value between all positive significant outcomes. The list of all the parameters, operators, and functions can be found on SAM's documentation website.

\section{The Workflow}

In order to describe the full process of conducting research shown in Fig 3 we will first outline a simple simulation scenario based on an one-way factorial design with two conditions where the Data Strategy is set to draw samples from a multi-variate normal distribution of $X \sim \mathcal{N}(\mu, \Sigma)$ with a mean vector of $\hat{\mu}=(0,0, x, x)$ and the covariance matrix of $\Sigma=I$. The Researcher is determined to find and report a significant outcome with a positive effect $(p$-value $<0.05 \&$ effect $>0$ ) and she is willing to report the alternative outcome variable, if necessary. Furthermore, she is particularity looking to submit an outcome with medium or larger effect size (effect $>0.5$ ). In fact, 276 
if the original study does not contain any desirable outcomes, she is planning to add 5 new samples to her data, and if that does not help, she is determined to remove the outliers of the dataset to achieve her goal. Our other player, the Journal, is configured to accept any upcoming Manuscripts and collects 24 publications from this scenario in order to study the biases that are introduced by Researcher actions'. To summarize, we are simulating a case where the Researcher is actively working to alter the Experiment and is willing to work her way toward significant and publication. See S2 File for the corresponding configuration file describing this simulation setup.

Starting from the top of the Fig 3 , our Researcher prepares the Experiment Setup (Fig $3[1-1]$ ), and based on its specification, she conducts the research and populates the Experiment with Data and other necessary statistics according to her methods of choice (Fig[3[1-2]). At this point where the Experiment is fully initialized, the Researcher can - and needs to - decide whether she wants to proceed to the publishing stage, or maybe try and apply a few of the QRPs to find a "better" outcome. This involves selecting outcomes from the Experiment (" $p$-value $<0.05$ \& effect $>0$ ") and deciding on whether she likes the outcome or not ("effect $>0.5$ "). Let's imagine that at this stage the Experiment does not contain any outcomes that satisfies our Researcher's criteria; therefore, she moves into the QRP section of the flowchart.

Except a few additional steps, this part of the process is very descriptive and straightforward. The Researcher decides whether she is going to start the process (Fig 3]2-2]), and from there she goes through her Hacking Strategies one by one, applies them (on top of each other) on the Experiment, performs a SDS (Fig[3]2-7], [2-8]) after each one, and decides whether she is satisfied with outcomes, or she perhaps prefers to go for a new method. Note that the user will be able to fine-tune the order, the technique of applying hacking-strategies by the Researcher, e.g., applying each strategy on a fresh copy of the Experiment instead of on top of the already altered Experiment.

One unusual step of this section is what we refer to as Stashing (Fig $3[2-6])$ where the Researcher gets to collect certain outcomes for later use. For instance, our Researcher may decide to collect all significant results that she finds along the way (either from the original or altered Experiment) in order to later search through them for "the best" outcome. (In our example, 304 our researcher is not configured to perform this step, and therefore the Stashed Outcomes Database is going to be empty, Fig[3]2-A])

After exhausting her list of $p$-hacking methods, our Researcher gets to replicate her Experiment and goes through the same routine again. This decision is based on two factors, 1) 
Fig 3. Research Workflow. The flowchart representation of the Research Workflow. The three main stages of the research process (introduced in Fig 1]and 2) are marked with numbers. The first two stages are performed by the Researcher while the last stage is done by the Journal. Here you can follow the footstep of the Researcher throughout the process of conducting her research. Notice how each Selection is followed by a Decision, i.e., every red rectangle is followed by a red diamond.

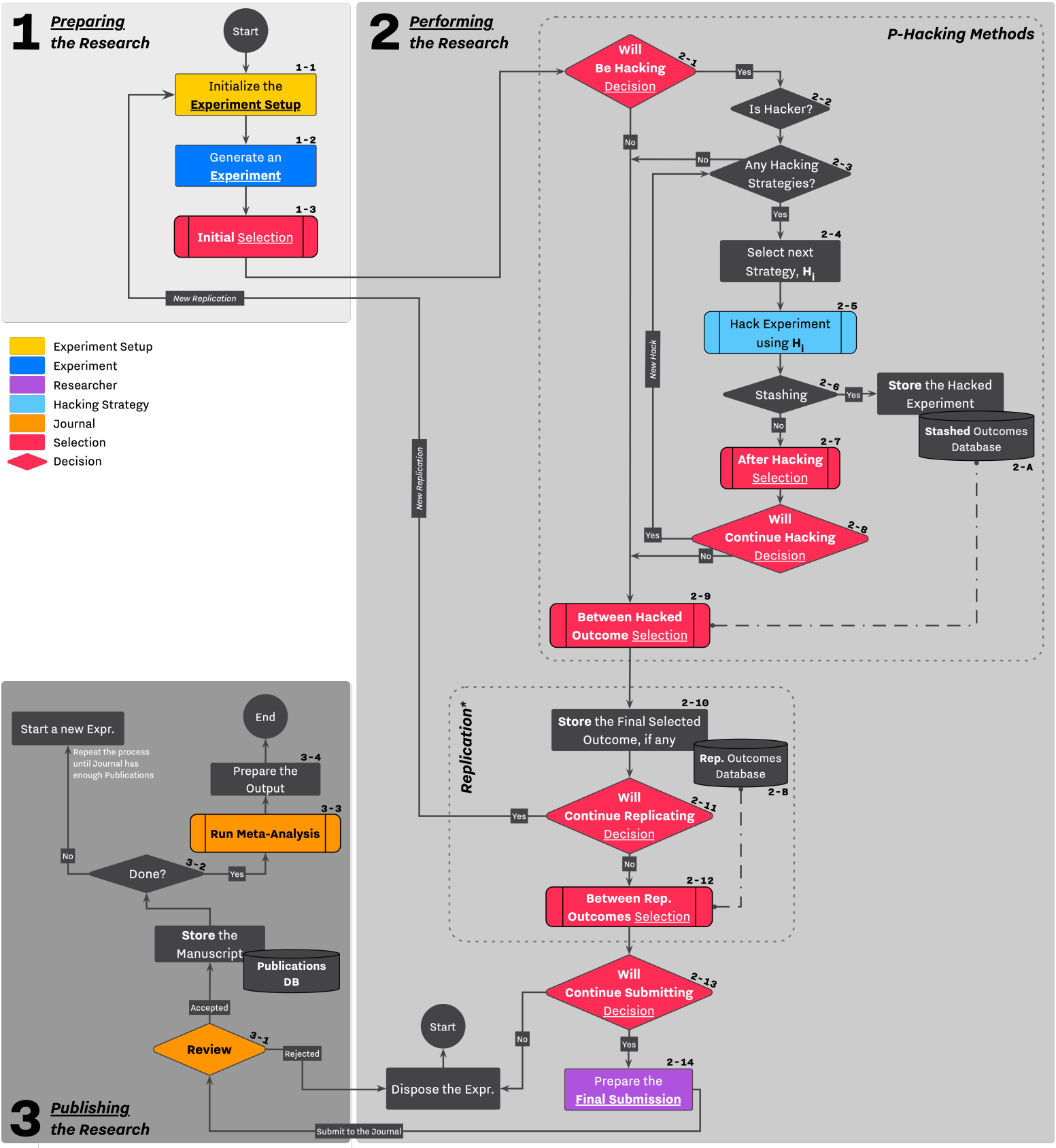

how many replications has she planned 2) whether she has already found a satisfactory outcome. 309 If any replications are carried on, the Researcher gets to collect outcomes from those 
[2-12]). However, if there are no replications planned, the Researcher arrives at her last decision where she is going to examine her Manuscript for the last time (Fig]3-13]) in order to decide on 313 whether she is going to submit it to the Journal or not. If the verdict is positive, she prepares a 314 Manuscript and sends it to the Journal. If not, the manuscript will be discarded, and the Researcher will start a new Experiment, i.e., back to Fig[3]-2].

The moment that the Manuscript is passed to the Journal, the Researcher cannot influence its destiny anymore, as we enter the last stage of the process. In this stage, the Journal evaluates the incoming Manuscript (Fig[3]-1]) and either accepts or rejects it. In our example, any Manuscript will be accepted regardless of its significance, i.e., no publication bias. After accepting 24 publications, the Journal calculates the random-effect estimate and report its estimate, as well as other relevant statistics to the user for further analysis.

\section{The Simulation}

One run of the simulation is finalized as soon as the Journal accepts its K'th publications. At this point, the Journal exports a list of 24 accepted publications in the form of a CSV file for further analysis by the user. In the case that we would like to repeat this scenario several times (to reduce the effect of randomness on our results), we may adjust the total number of iterations in the Simulation Module, as described in the previous section. As a result, SAM repeats the current simulation setup $\mathrm{N}$ more times and export $24 \times \mathrm{N}$ publications in the batches of 24 for further analysis. At the end of the simulation procedure, SAM produces several output files that may be used with any programming language or analysis toolkit for further analysis. Note that the user can change the type of information that is going to be exported at the end of the simulation. For instance, he/she can even configure SAM to report the list of all rejected Manuscripts in order to study a specific metric, e.g., evaluating the success of Journal's Review Strategy in rejecting biased studies.

\section{The User's Perspective}

As we discussed, the user of SAM is in control of the entire simulation scenario. In the case above, we, as the user, configured every module according to our sample simulation design. Note that at any point, we were able to add, remove or modify the details of each module, and therefore model a new scenario, or study the influence of an alternation on our design. For instance, we could have replaced the Research Strategy with a more honest approach where the Researcher only reports 
the pre-registered outcome. This would have allowed us to implement a control simulation

scenario. The same is true when it comes to the Journal module, we could have replaced the Review Strategy to induce a certain level of the publication bias in order to study the accumulative effect of our model in the presence of publication bias. In fact, this is what we refer to as flexibility and modularity. From users' perspective, the only thing that needs to be changed in order to add or remove new dimensions to the simulation is adding, removing or modifying individual modules.

\section{Getting Started with SAM}

The S3 Appendix contains a short introduction on how to install, and configure SAM in our system, and experiment with a sample simulation setup. In addition, please visit our website to get the latest information and tutorials.

\section{Results}

In this section, we illustrate how SAM can be used to reproduce a fairly complicated simulation study of Bakker et al. 32. In the first part, we capitalize on the flexibility and modularity of SAM to reproduce and dissect the original simulation study in order to isolate different processes and factors of the design. This helps us reveal the true sources of the biases captured in the original study. In the second part, we extend the original simulation by introducing new modules and processes into the design in order to study the influence of smaller $\alpha$, more aggressive $p$-hacking procedures, and the publication bias on the effect size bias and the power of Egger's Test.

\section{The Simulation Setup}

As described by Bakker et al. [32], their simulation study is investigating the four following scenarios. Note that in the original text, Bakker et al. refer to these cases as strategies; however, 362 in this text, we will refer to them as "scenarios" to mitigate confusion with other SAM's strategies. 363

Scenario 1. Perform one large study (with $\mathrm{N}$ as the sample size) with sufficient 
power and publish it.

Scenario 2. Perform one large study and use some of the QRPS most popular in psychology [29]. These QRPs may be performed sequentially until a significant result is found:

- Test a second dependent variable that is correlated with the primary dependent variable (for which John et al. found a $65 \%$ admittance rate)

- Add 10 subjects (sequential testing; $57 \%$ admittance rate)

- Remove outliers $(|Z|>2)$ and rerun analysis (41\% admittance rate)

Scenario 3. Perform, at most, five small studies each with (N/5) as sample size. Players may stop data collection when they find a significant result in the expected direction and only publish the desired result (the other studies are denoted "failed"; $48 \%$ admittance rate).

Scenario 4. Perform, at most, five small studies and apply the QRPs described above in each of these small studies if the need arises. Players may report only the first study that "worked."

- Bakker, van Dijk, and Wicherts [32]

In order to reproduce this simulation using SAM, we first need to configure each module according to the specifications of Bakker et al. Starting from the Experiment Setup, in all scenarios, the Researcher works with a one-way factorial design with two conditions, one with small sample sizes $\{5,10,20\}$, and another one with large sample sizes $\{25,50,100\}$. The measurements are drawn from a multivariate normal distribution, i.e., the Data Strategy, with a specific covariance matrix enforcing 0.5 correlation between outcomes. Researchers use $T$-Test as their Test Strategy and Standardized Mean Differences as their Effect Strategy. This is shown in the first panel of the Fig 4, Preparing Research. In addition, Bakker et al. indicate that Researchers may use up to three different Hacking Strategies, and they may choose different dependent variables as their final outcome, i.e., Manuscript, as listed in the middle panel of the Fig 4. Performing Research. Finally, the Journal accepts any incoming Manuscripts, and it stores a list of publications for further analysis as listed in the right panel of the Fig 4 , Publishing 
Fig 4. The Simulation Setup of Bakker et al. The representation of SAM's components and research process adapted to the specifications of the simulation study of Bakker et al. The details of the Research Strategy/Workflow is discussed in Fig 5 . The labeled elements $(\mathbf{A}, \mathbf{B}, \ldots)$ indicate the modules that will be modified in order to extend the original simulation in the following sections.

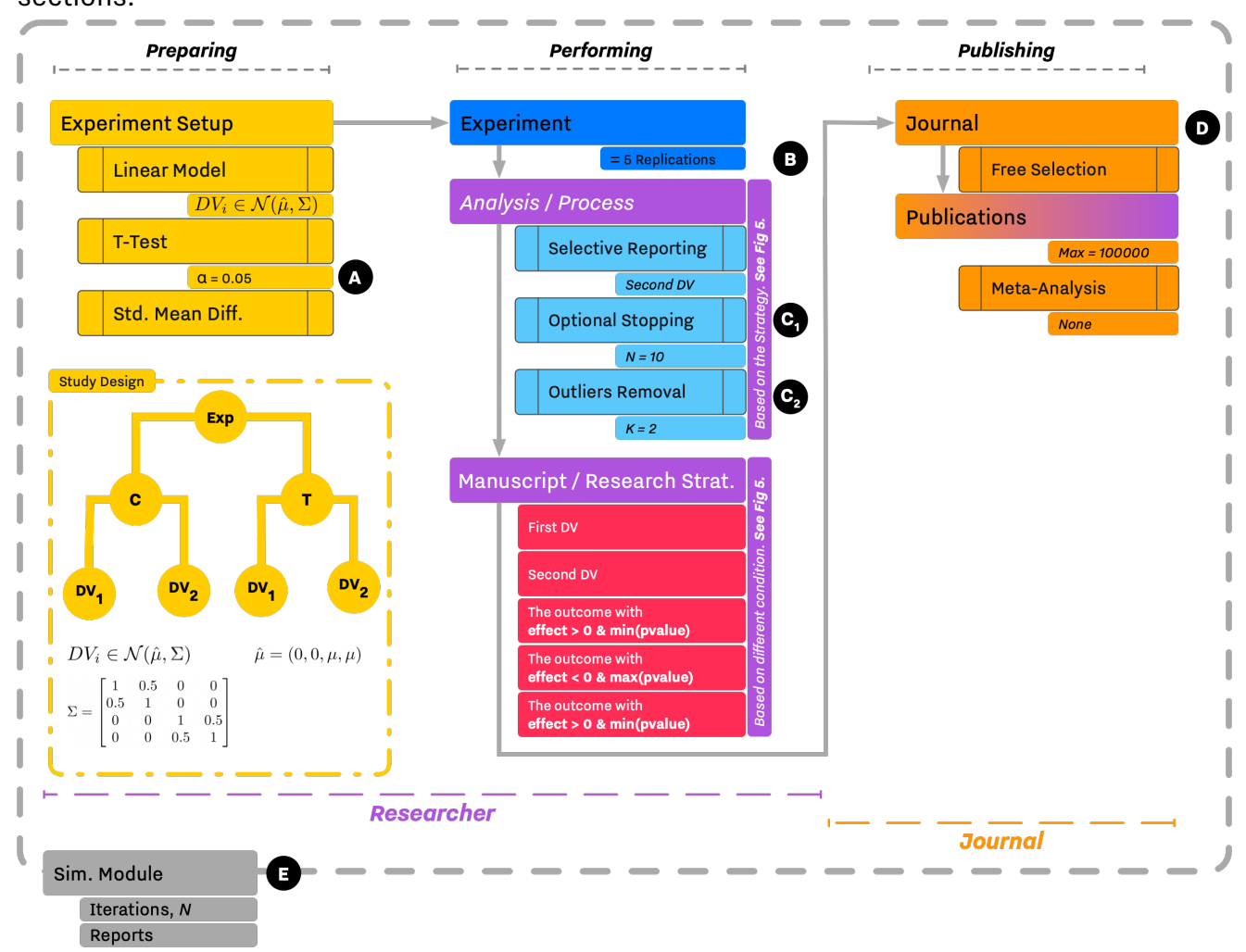

While Fig 4 shows the overall configurations of each module, we still need to know how the Research Strategy is specified for each scenario. Fig 5 is constructed based on the simulation specifications and provided R code by Bakker et al. As shown, the Researcher needs to perform a series of selection-decision sequences, SDS, in order to find a "desirable" outcome before heading to the Journal. Within the $1^{\text {st }}$ scenario, the Researcher performs no extra actions on the Experiment and simply returns the first dependent variable, i.e., control simulation. In all the other scenarios, the Researcher will attempt to "hack" her way toward significance if she is unable find a "desirable" result, in this case, an outcome with " $p$-value $<0.05$ \& effect $>0$ ". Additionally, the Researcher repeats the same Experiment up to 5 times, stashes her findings and selects the best outcome between them. Fig 5 clarifies these attempts by showcasing how a 
Fig 5. The Simulation Flow of Bakker et al. Left Panel: The flowchart representation of the $1^{\text {st }}$ and $2^{\text {nd }}$ scenarios. Right Panel: The flowchart representation of the $2^{\text {rd }}$ and $4^{\text {th }}$ scenarios. In the $1^{\text {nd }}$ and $3^{\text {rd }}$ scenarios, the simulation skips the highlighted QRP Procedure; however, in $3^{\text {rd }}$ scenario where the Researcher set to run 5 replications, she runs 5 replications (without hacking) and collects the "best" outcome of each study, i.e., Stashing; and finally, she searches the database and select the "best" results in order to construct her final Manuscript for the submission. Read more on Bakker et al. [32].

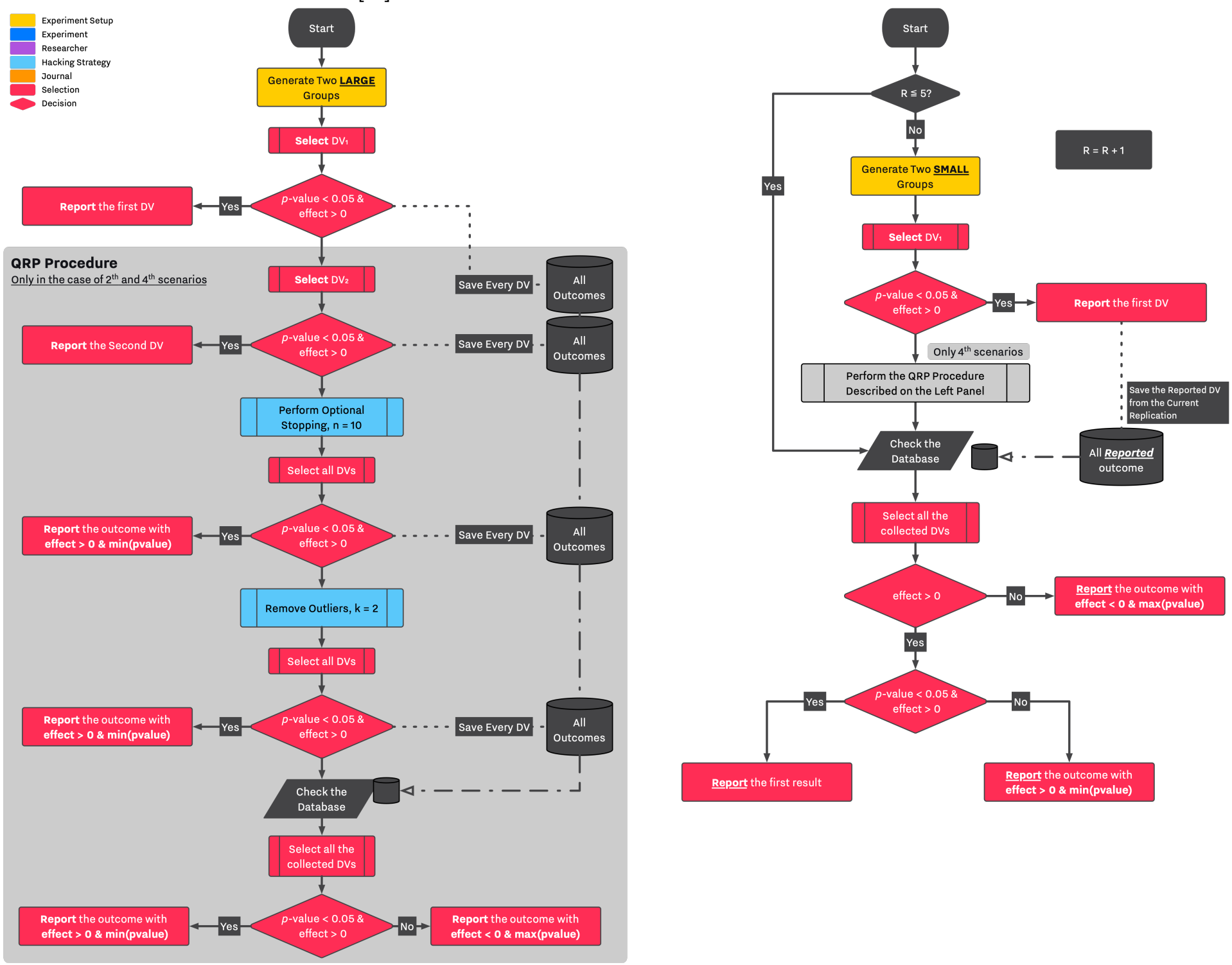

In summary, the simulation study of Bakker et al. consists of two distinct Experiment Setups and four different Researchers each with their own unique Researcher Strategies. The modularity of SAM allows us to independently configure these modules, and later use them as building blocks 
to construct either of the scenarios. Fig 6 shows a sample configuration used to model the $4^{\text {th }}$

scenario. As mentioned, there are three main components to configure, the experiment

parameters are describing the small study design of $4^{\text {th }}$ scenario and are defining the scope of

the Experiment. The researcher parameters are configuring the behavior of the Researcher

according to the right flowchart shown in Fig 5 . Notice the chain of selection-decision sequences

that are translating the complicated design of Bakker et al. into a set of simple logical rules that

defines the behavior of the Researcher. Finally, the journal parameters are creating a Journal

that accepts 10000 publications.

Fig 6. Sample SAM's Configuration for Scenario 4 of the Simulation Study of Bakker et al.

Notice the path and definition of various selection-decision sequences describing the Research Strategy of Bakker et al. according to Fig 4 and 5 . The labeled elements $(\mathbf{A}, \mathbf{B}, \ldots)$ indicate the modules that will be modified in order to extend the original simulation in the following sections.

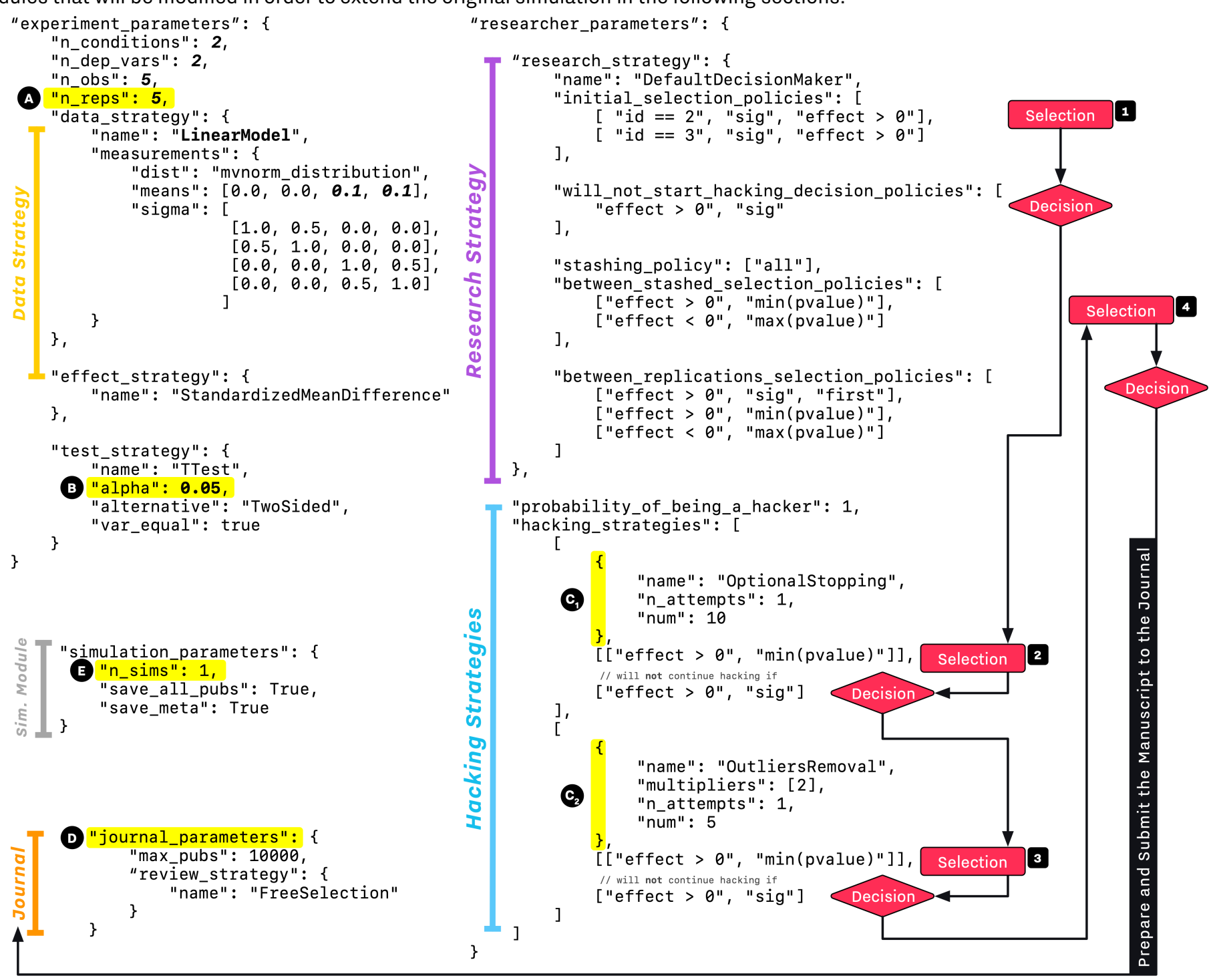


As soon as we have the main body of the configuration ready, if needed, we can simply modify a module without affecting others, e.g., modify one of the $p$-hacking methods. Similarly, if we need to run the same setup with different parameters, we only need to adjust the target parameter and repeat the simulation. For instance, in order to run each scenario with different true effect sizes, we only need to change the means vector of the multivariate normal distribution. In the following sections, we are going to use this approach in order to reproduce and extend the simulation study of Bakker et al. We use Fig 4 and 6 as templates and showcase how simple modifications to each part of the design and configuration file can extend the simulation.

The reproducible code for all the following simulations, results, and visualizations can be found on S4 File.

\section{The Reproduced Simulation}

Our first attempt to reproduce the results of Bakker et al. revealed a minor bug in their code that resulted in them capturing less bias in the fourth scenario (see S1 Fig). After correcting and rerunning their code, we achieved a good fit between our results and their results, as shown in Fig]7. We have used a similar visualization to the Fig 2 of Bakker et al., and we will use this arrangement throughout the rest of the paper. This entails that, in all our plots, green lines correspond to $2^{\text {nd }}$ and $4^{\text {th }}$ scenarios, where QRPs were applied on the Experiment; and, blue lines correspond to $1^{\text {st }}$ and $3^{\text {rd }}$ scenarios where no QRPs were applied by the Researcher. The solid lines represent Large studies, and dashed lines represent Small studies; and each row correspond to one pair of sample size. E.g., $\mathbf{S} ; \mathbf{N}=10$ indicates that Large studies of $1^{\text {st }}$ and $2^{\text {nd }}$ scenarios used $\mathbf{N}$ $=\mathbf{5 0}(\mathbf{5} \times \mathbf{1 0})$, and Small studies of $3^{\text {rd }}$ and $4^{\text {th }}$ used $\mathbf{N}=\mathbf{1 0}$ observations per DV. Finally, the $\mathrm{x}$-axis shows a full range of true effect sizes $\{0.0,0.1,0.2,0.3,0.4,0.5,0.6,0.7,0.8,0.9,1.0\}$, and $y$-axis (Bias) indicates the difference between the true effect size, and the calculated standardized mean differences. See Bakker, van Dijk, and Wicherts [32] for more info.

\section{Extensions}

After successful replication of the original simulation, we can now leverage on the modularity and flexibility of SAM and extend the simulation study of Bakker et al. [32]. The modularity of SAM should allow us to easily interchange or modify different modules, and consequently able us to re-run the simulation without worrying about other components. Here, we are going to experiment 
Fig 7. Comparison Between the Original and Reproduced Simulation. Each plot showcases 4 different results. Green lines correspond to $2^{\text {nd }}$ and $4^{\text {th }}$ scenarios, where QRPs were applied on the Experiment; and, blue lines correspond to $1^{\text {st }}$ and $3^{\text {rd }}$ scenarios where no QRPs were applied by the Researcher. The solid lines represent Large studies, and dashed lines represent Small studies; and each row correspond to one pair of sample size. E.g., $\mathbf{S} ; \mathbf{N}=\mathbf{1 0}$ indicates that Large studies of $1^{\text {st }}$ and $2^{\text {nd }}$ scenarios used $\mathbf{N}=\mathbf{5 0}(\mathbf{5} \times \mathbf{1 0})$, and Small studies of $3^{\text {rd }}$ and $4^{\text {th }}$ used $\mathbf{N}=10$ observations per DV.

Chance of Finding sig.

$\alpha=0.05$
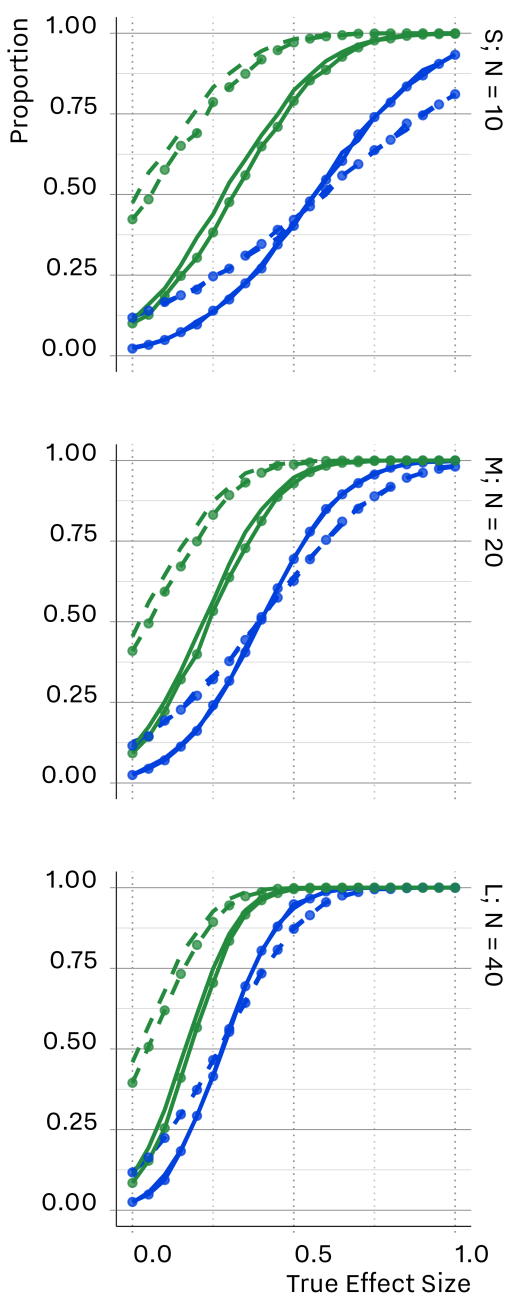

\section{Effect Size Bias}

$\alpha=0.05$
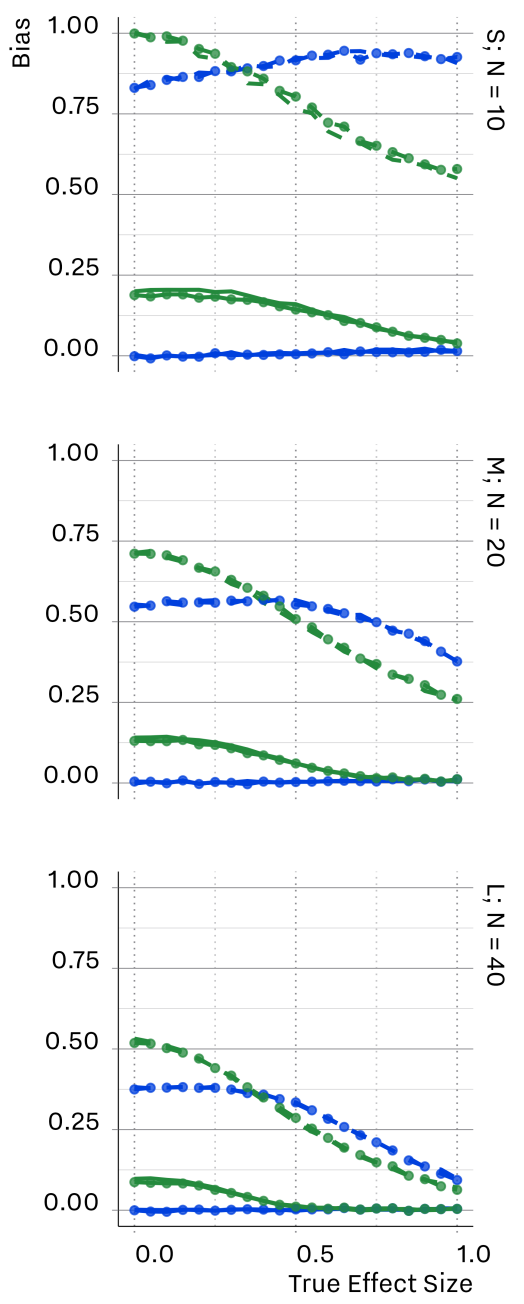

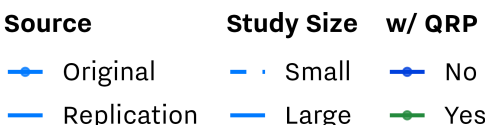




\section{Different Significance Levels}

In our first extension, we aim to address a controversial question of whether lowering the $\alpha$ mitigates the influence of the processes deployed by Bakker et al. [32]. In order to answer this question, we need to run our simulation with different $\alpha$ 's. This can easily be done by adjusting the $\alpha$ of the Test Strategy and leaving everything else intact, labeled $\mathbf{A}$ in Fig 4 and 6 . Fig 8 shows the level of effect size bias for three different $\alpha$ 's, $\{0.0005,0.005,0.05\}$. As we can see, the effect of lowering $\alpha$ on effect size bias is not as drastic or consistent as one would have expected [35]. In fact, lowering $\alpha$ does not necessary reduce the effect size bias; in contrast, in all scenarios and configurations, the smaller $\alpha$ leads to slightly higher effect size bias. In addition, the loss in power is evident from the lower chances of finding significant results. Also, interesting to note that the small sample strategy appears to be less effective under lower alpha levels, which is positive, as it may imply that fewer researchers would opt for smaller samples under lower alpha levels. This is to be expected given the larger power requirements under lower alpha values.

\section{Effects of Replications}

In addition to a series of QRPS, Bakker's Researcher also take advantages of Stashing across the hacked and replications results, in $2^{\text {nd }}, 3^{\text {rd }}$, and $4^{\text {th }}$ scenarios. This gives the Researcher a great deal of freedom to choose between different paths, procedures and outcomes throughout the entire process, see Fig 4 and Fig 5 . In order to differentiate between the influence of the replication and stashing procedure, and the impact of questionable research practices, we ran 5 new scenarios in which Researchers were allowed to perform exactly $\mathrm{R}$ replications, $\{1,2,3,4,5\}$. This can easily be done by adjusting the number of replications, labeled $\mathbf{B}$ in Fig 4 and 6 .

As shown in Fig 9 , in all the scenarios, the severity of bias consistently increases as we increase the maximum number of replications allowed. In addition, we can observe how different number of replications interacts with the level of $\alpha$ as well as the true effect size. In fact, we can see how using more replications increases the effect size bias as we lower the $\alpha$, especially as we move toward larger true effect sizes. Furthermore, we can see the isolated effect of $p$-hacking methods deployed by Bakker et al. by studying the first column. In all the cases, the notable difference between results from 1 and 5 replications suggests that the main sources of the biases reported by Bakker et al., were in fact from the replication procedure; and p-hacking's effects are 
Fig 8. Influence of Different $\alpha$ Levels on Proportion of Significant Results, and the Effect Size

Bias. Each column represents different level of test $\alpha,\{0.0005,0.005,0.05\}$, respectively from left to right.

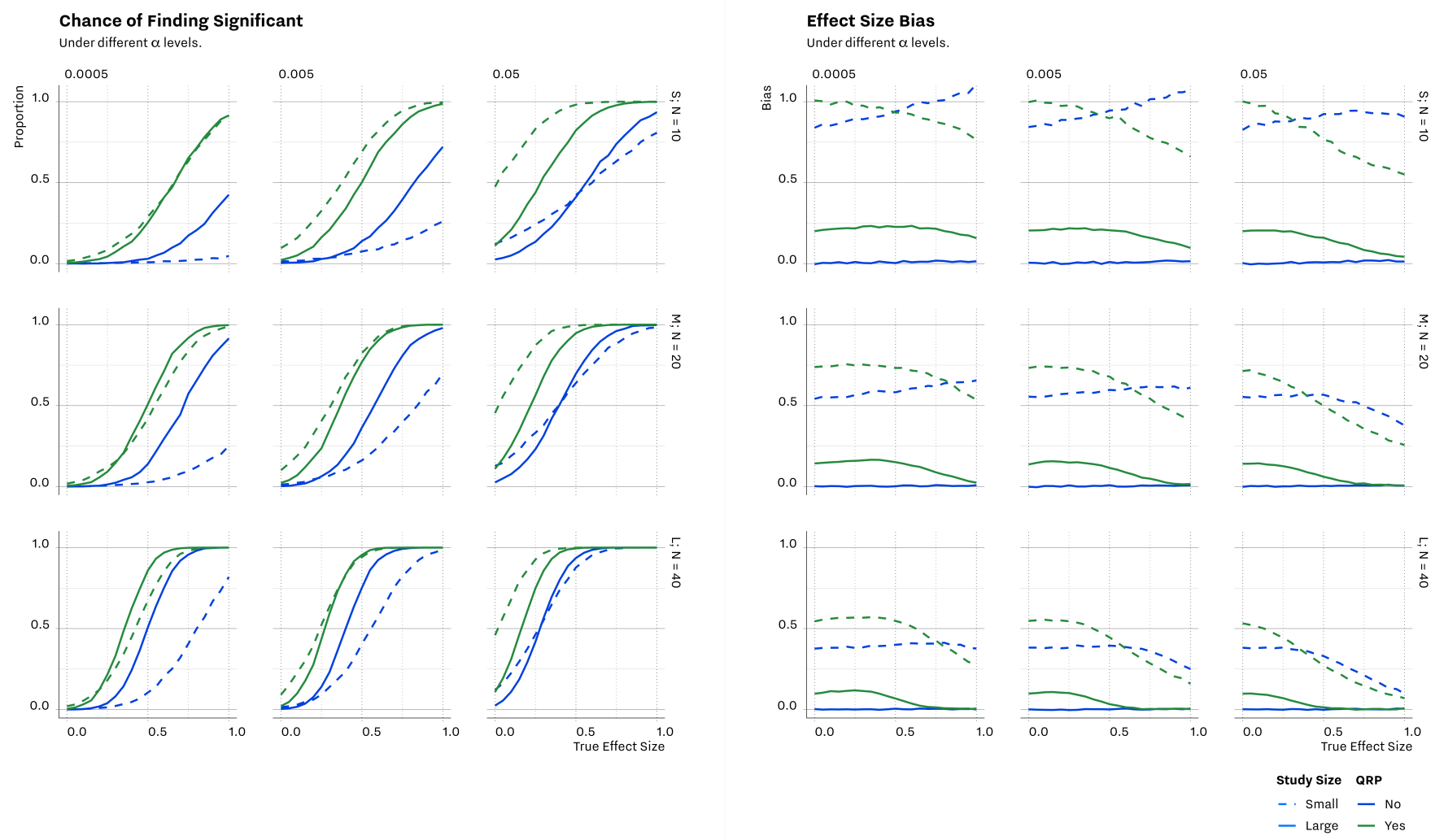

only responsible for maximum half of the bias. In other words, the main contributor to the effect size bias is the process of selecting between a collection of replicated/hacked outcome variables.

In fact, this observation strengthens the hypothesis and conclusion drawn by Bakker et al. by showcasing the immense effect and advantage of running small studies over larger studies. Studies with low sample size are volatile enough that researchers could easily achieve significant results with unrealistic effect sizes across the true effect size spectrum only by repetition.

\section{More Aggressive QRPs}

In order to further investigate the effect of QRPS, we decided to take advantages of SAM'S modularity, and replace the two existing QRPs, with more aggressive versions of them, e.g., Adaptive Optional Stopping, and Subjective Outliers Removal [36]. Within the new configuration, our Researcher adapts its optional stopping by adding $1 / 3 \times N$ new observations to every DV of the Experiment (instead of a fixed value, $n=10$ ); moreover, she gradually reduces the threshold of

(1)


Fig 9. Influence of Different Number of Replications on Effect Size Bias. Each column represents different number of replications performed by the Researcher, 1, 2, 3, 4, 5, respectively from left to right.

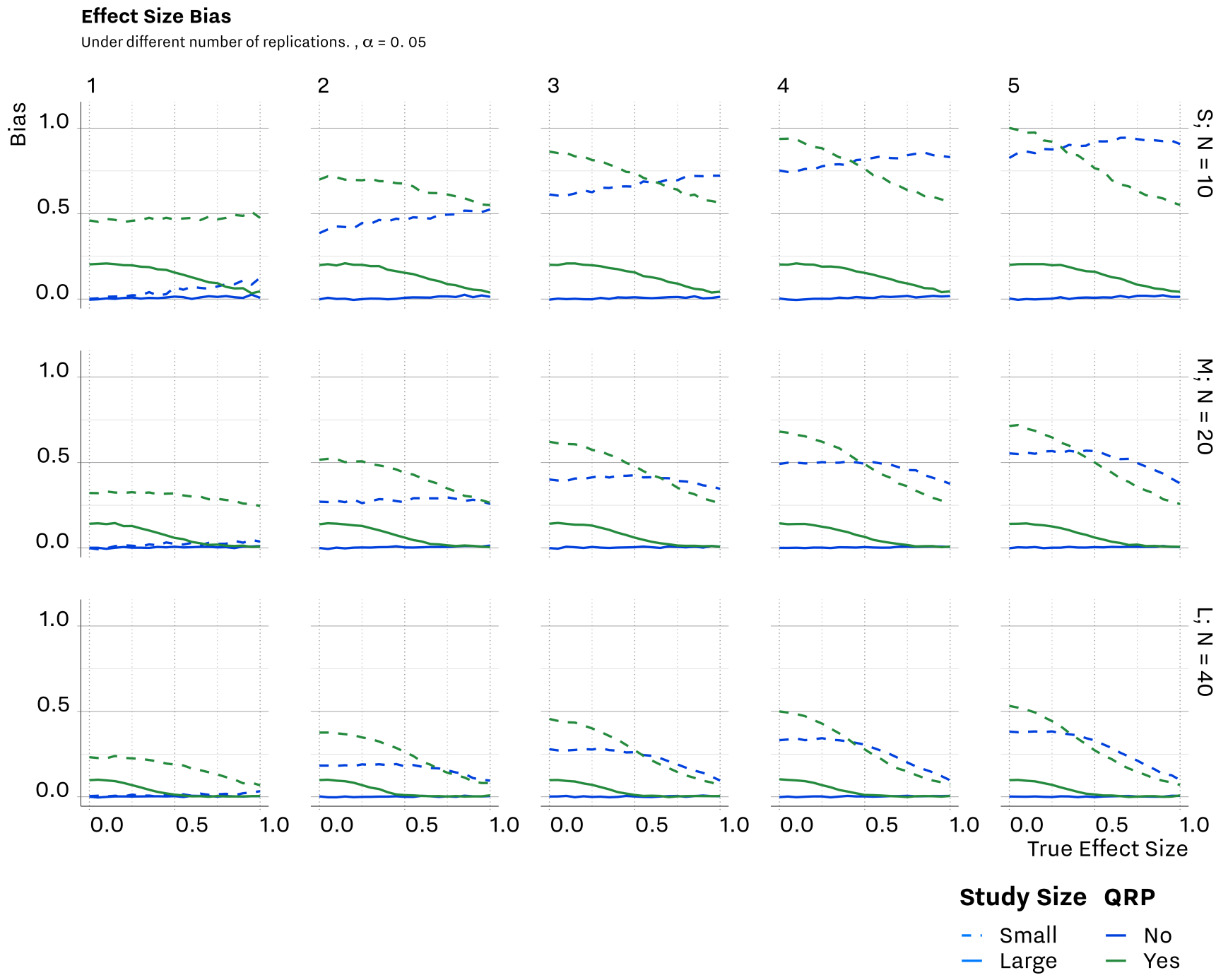

searching for outliers from 4 to $2,\{4,3.5,3,2.5,2\}$, and stops as soon as she finds a significant result. This can be done by replacing modules labeled $\mathbf{C}_{\mathbf{1}}$ and $\mathbf{C}_{\mathbf{2}}$ in Fig 4 and 6 with snippets of Listing 1 Notice the stopping condition parameters which is used by the user to stop the Outliers Removal as soon as one of the outcome variables became significant.

Listing 1. Snippets for More Aggressive Hacking Strategies

$$
\begin{aligned}
& \{/ / \mathrm{C} 1 \\
& \text { "name": "Optionalstopping", }
\end{aligned}
$$




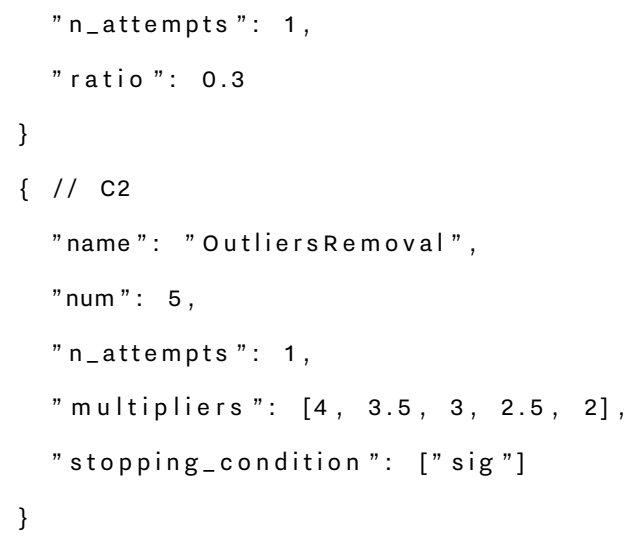

As shown in Fig 10, there is a significant difference between the level of bias between the p-hacking setup of Bakker et al., and our more aggressive setup. However, the contrast between two setup shrinks as soon as we move to higher powered studies. In fact, in high-powered studies, i.e., the last row, there is little to no difference between the level of bias between the two setups. Also, note the effect of lower $\alpha$ on the bias, in all cases, as we move toward lower $\alpha$ 's, we see more bias.

\section{Publication Bias and Egger's Test Power}

Bakker et al. have not introduced any publication bias at the level of journal in their simulation. Here, in our last extension, we decided to modify the Journal module by replacing its Review Strategy with an algorithm that administers different levels of publication bias when reviewing incoming Manuscripts.

As listed in Table 1, the "Significant Based Review Strategy" accepts any significant incoming Manuscripts, and if they are not significant, it will give them $\left(1-P_{b}\right)$ chance of being accepted, therefore forcing $\mathrm{P}_{\mathrm{b}}$ publication bias. In addition to applying the publication bias to Journal's Review Strategy, we are going to assign a publication bias measure to the Journal, e.g., Egger's Test. Finally in order run the test on a reasonable number of studies, we limit the maximum number of Journal's publications to max_pubs $=24$. Like our other extensions, we do not need to modify any other parts of the simulation; in fact, running the simulation with the Journal's configuration of Listing 2 will be sufficient, labeled $\mathbf{D}$ in Fig 4 and 6 .

Listing 2. Snippets for Journal's Configuration

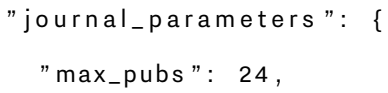


Fig 10. Influence of More Aggressive QRPs on Effect Size Bias. The influence of more aggressive questionable research practices on the effect size bias. In this case, the Researcher performs an optional stopping by adding $1 / 3 \times N$ new observations to each DV, and removing outliers while reducing $\mathrm{K}$ until she finds a significant result.

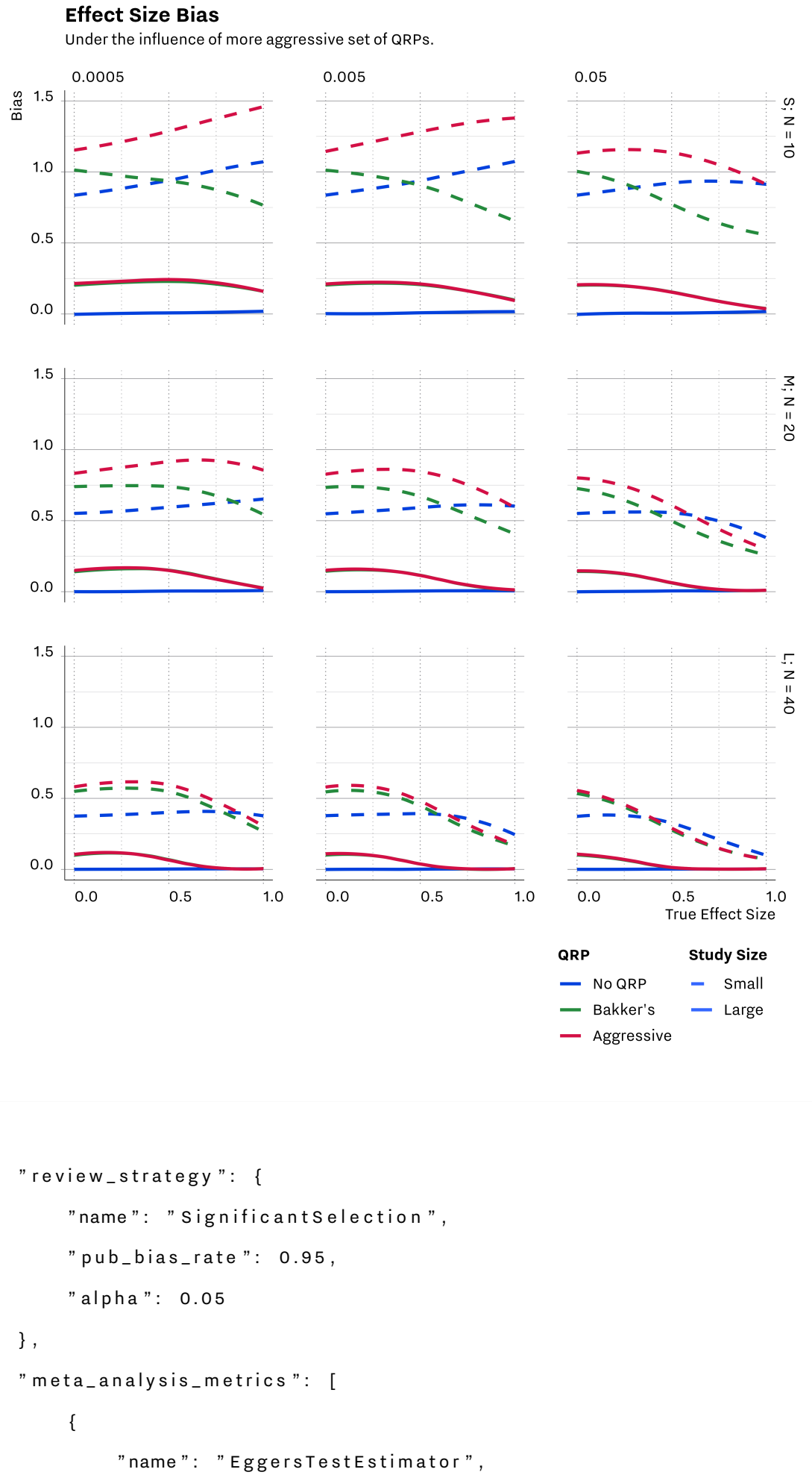


It is worth noting that now that the Journal collects only 24 publications, we need to increase the number of simulation cycles to 10000 in the simulation Module, labeled $\mathbf{E}$ in Fig 4 and 6 . By increasing the number of iterations to 10000 , the Journal will report a total of 240,000 records in batches of 24 publications. In this way, every batch of output contains the $p$-value of the Egger's test as well as a list of publications and their statistics necessary for post-analysis and visualization.

Fig 11 shows the effect of publication bias on the level of effect size bias. On the left panel, we see the effect of publication bias, on scenarios with no QRPs $\left(1^{\text {st }}\right.$ and $3^{\text {rd }}$ scenarios $)$. Notice the extreme level of bias as we start increasing the publication bias rate (darker the lines, higher the $\mathrm{P}_{\mathrm{b}}$ ) especially among the lower-powered studies of the first row. This combined with the effect of lowering $\alpha$, as seen previously, leads to a detrimental level of bias in small-sized studies. In contrast, the level of bias on the right panel, studies with QRPs ( $2^{\text {nd }}$ and $4^{\text {th }}$ scenarios), is not as high as the left panel. This is most likely due to addition of 10 new observations to every DV by the optional stopping.

Fig 12 shows the effect of publication bias on the power of Egger's publication bias test. On the left panel, we observe a relatively poor and inconsistent performance of the test. This is especially notable in low-powered and small-sized studies (dashed blue lines of the first row) where in lower $\alpha$-regimes, Egger's test confidently reports at least $50 \%$ publication bias even in the cases were we have not introduced significant publication bias (brighter dashed line). While the trends are slightly different on the right panel, we can at least observe a more consistent performance (overlapping lines). Note that the current simulation design uses very similar sample sizes across studies in a meta-analysis. Hence, the small variation poses a demanding scenario for detection methods like Egger's test that specifically test for moderation of effects by sample size or standard error. We expect Egger's test to perform better when sample sizes vary over studies, as they would in most meta-analyses 
Fig 11. Influence of Publication Bias on Effect Size Bias. The darker the lines the more publication bias is induced by the Journal during the review process, see legend. Left Panel: studies with no QRPs (blue lines). Right Panel: Studies with QRPs from Bakker et al. (green lines).
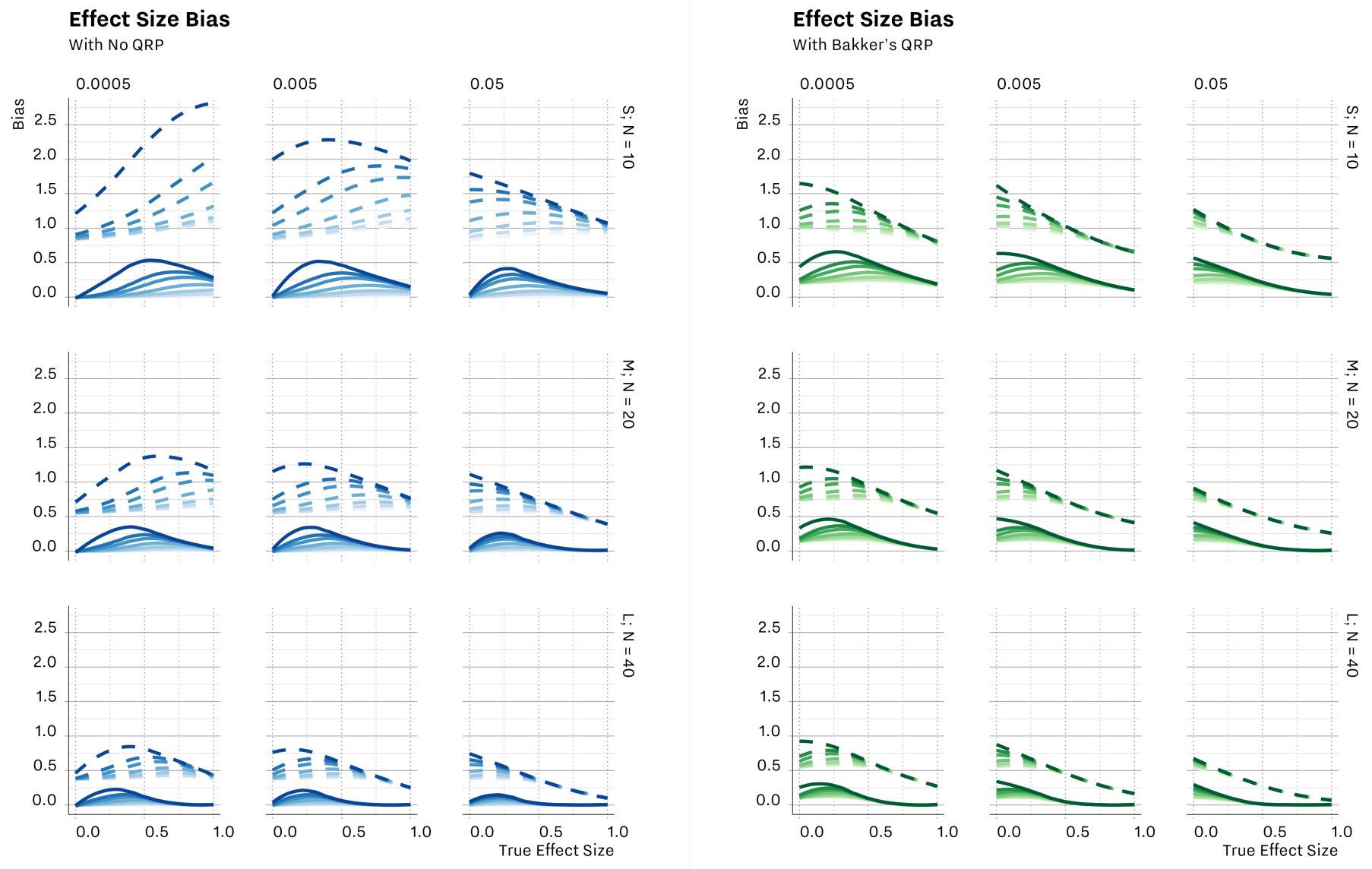

\section{Discussions}
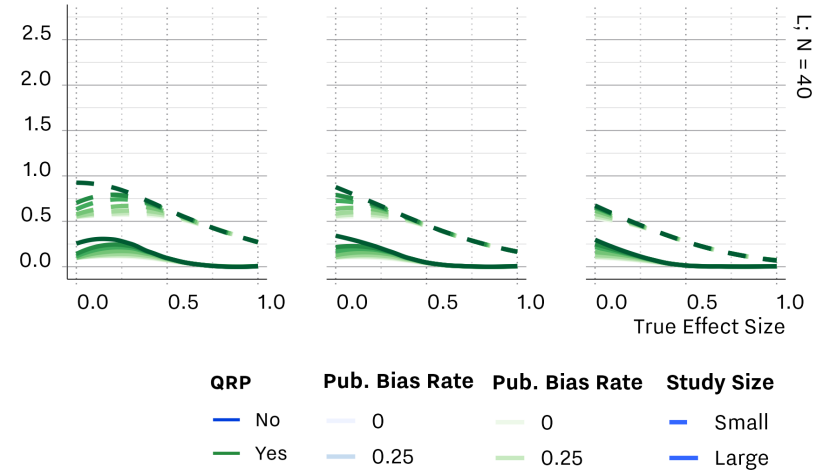

$\begin{array}{ccc}\text { Pub. Bias Rate } & \text { Pub. Bias Rate } & \text { Study Size } \\ 0 & 0 & -\quad \text { Small }\end{array}$

$0.25-0.25 \quad-$ Large
$-0.5-0.5$

$-0.5 \quad-0.5$

$-0.75-0.75$

$-0.9-0.9$
-0.95

$-0.95-0.95$

$-0.99-0.99$

In this paper, we introduced and described an abstract representation of the process of conducting research. We explored this process in detail, and exposed its sub-processes and components. We discussed how the complexity of individual components combined with the intertwined nature of their interactions as well as a series of intricate decision-making routines 
Fig 12. Influence of Publication Bias Rate on Egger's Test Power. The darker the lines the more publication bias is induced by the Journal during the review process, see legend. Left Panel: studies with no QRPs (blue lines). Right Panel: Studies with QRPs from Bakker et al. (green lines).
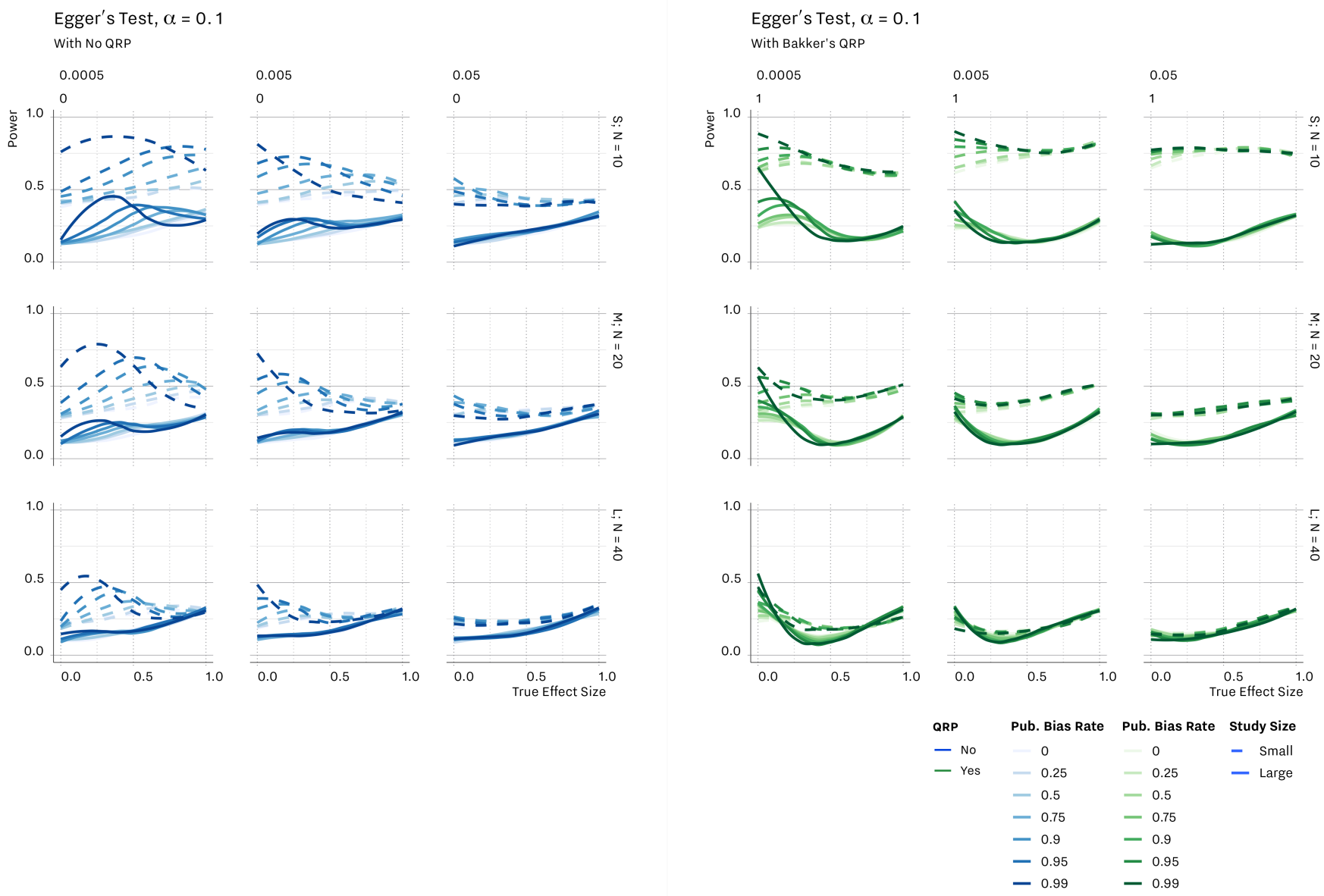

ultimately create a complex system [37]. We argued that in order to track down roots of the biases

that are affecting our scientific findings we need to analyze the coexistence and interactions between these components and processes. Finally, we introduced our versatile simulation framework, SAM, and argued that its ability to model the process of conducting research in a modular manner will help us to systematically study the complexity of this process and unravel true sources of the biases affecting our research findings.

In the last section, we demonstrated the procedure of reproducing an existing simulation study using SAM. Our independent reproduction process was able to correctly reproduce and even uncover a minor bug in the original simulation. In addition, while we did not set to conduct a full-fledged simulation study, we showcased the power and versatility of SAM - at modifying, 
adding or removing simulation elements- by building on top of the simulation study of Bakker et

al. Our back-of-the-envelope demonstrative simulation study suggests that the idea of lowering $\alpha$ may not be as effective as we hope it to be; in fact, we could not observe a consistence reduction in effect size bias within different $\alpha$-regimes.

As we pointed out throughout this work, one simulation study will not be able to do the question in this caliber justice. In order to systematically address such a complicated question, we need to consider numerous factors, e.g., the influence of different data models and test statistics, the effect of variety of researchers' degrees of freedom, the role of publication bias in conjunction with researchers' degrees of freedom, and finally the collective impact of all these factors on our scientific findings. It is only then that we may be able to formulate some preliminary conclusions on whether lowering $\alpha$-level might in fact address part of our collective problem. The same goes for investigating the extent of Bayesian approaches' effectiveness; similarly, one needs to consider various factors before coming to a meaningful conclusion.

In fact, as we illustrated, SAM is designed and developed to precisely address this complexity and variability by offering three primary qualities: modularity, flexibly and extensibility. The Modularity allows for seamless interchangeability of modules and guarantees their compatibility. The Flexibility makes it possible for every module to be configured individually before being placed in the process. Finally, the Extensibility facilitates the path for implementing new internal modules and components, and integration of SAM into other frameworks as an add-on. We believe that these design objectives combined with our extensive effort to illustrate the process of conducting research as closely as possible have resulted in SAM being a versatile simulation framework that is capable of modeling a wide range of simulations and scenarios.

In fields like psychology - and simulation studies like Bakker et al. - where every step and decision are revolved around statistical testing, SAM's extensive list of methods and algorithm can be used to model different Researcher's behavior as well as Journal's policies. We believe these methods could help us to better track and understand the biases resulted from researchers' questionable research practices and/or journals' questionable reviewing policies.

\section{Future Development}

Our main priority is to keep the codebase and its documentation alive and up to date, as well as improving the usability and friendliness of our toolset. In addition, we continue implementing new 
algorithms and strategies to the main framework. We are hoping that with the help of other

meta-scientists, we will be able to grow our library, and overcome some of the challenges

regarding the research and implementation of some of the highly controversial and sophisticated

methods, e.g., Falsifying Data and Fabricating Data Hacking Strategies. Furthermore, we believe

the availability of SAM's Core APIs should facilitate the process of extending SAM into other

programming languages, e.g., R. We envision a GUI, Graphic User Interface, for SAM that may

allow the user to visually put the pieces of his simulation together, design the research process

and observe Researcher's decisions and track biases in real-time.

\section{Supporting Information}

S1 Fig. Comparison between the un-patched results from Bakker et al. and SAM's output. As shown, there is a small discrepancy between outcomes of Bakker et al. and SAM's outputs. This is due to a minor bug in the simulation study of Bakker et al. which led to capturing lower bias in Scenario 4 of their simulation.

S2 File. Configuration File for the Sample Research Workflow. SAM's configuration file corresponding to the sample simulation described in Fig 3 .

S3 Appendix. Getting Started with SAM. A short installation and tutorial guide on how to get started with SAM. Find the complete tutorial on our website.

S4 File. SAM's Simulation Source and Results. An archive file containing SAM's source code, $\quad 607$ and all the reported results, and figures. For the updated simulation material, please visit: $\quad 608$ https://github.com/amirmasoudabdol/bakker-et-al-2012-reproduction-using-sam

\section{Funding}

The preparation of this article was supported by an ERC consolidator grant IMPROVE (grant no. 


\section{References}

1. Ioannidis JP, Fanelli D, Dunne DD, Goodman SN. Meta-research: Evaluation and Improvement of Research Methods and Practices. PLoS Biol. 2015;13(10):e1002264. doi:10.1371/journal.pbio.1002264.

2. Wicherts JM, Veldkamp CLS, Augusteijn HEM, Bakker M, van Aert RCM, van Assen MALM. Degrees of Freedom in Planning, Running, Analyzing, and Reporting Psychological Studies: A Checklist to Avoid p-Hacking. Frontiers in Psychology. 2016;7(1832). doi:10.3389/fpsyg.2016.01832.

3. Simmons JP, Nelson LD, Simonsohn U. False-Positive Psychology: Undisclosed Flexibility in Data Collection and Analysis Allows Presenting Anything as Significant. Psychological Science. 2011;22(11):1359-1366. doi:10.1177/0956797611417632.

4. van Aert RC, Wicherts JM, van Assen MA. Conducting meta-analyses based on $p$ values: Reservations and recommendations for applying $\mathrm{p}$-uniform and $\mathrm{p}$-curve. Perspectives on Psychological Science. 2016;11(5):713-729.

5. Carter EC, Schönbrodt FD, Gervais WM, Hilgard J. Correcting for bias in psychology: A comparison of meta-analytic methods. Advances in Methods and Practices in Psychological Science. 2019;2(2):115-144.

6. McShane BB, Böckenholt U, Hansen KT. Adjusting for publication bias in meta-analysis: An evaluation of selection methods and some cautionary notes. Perspectives on Psychological Science. 2016;11(5):730-749.

7. Nosek BA, Beck ED, Campbell L, Flake JK, Hardwicke TE, Mellor DT, et al. Preregistration is hard, and worthwhile. Trends in cognitive sciences. 2019;23(10):815-818.

8. Gronau QF, Duizer M, Bakker M, Wagenmakers EJ, Gronau QF. Bayesian Mixture Modeling of Significant P Values: A Meta-Analytic Method to Estimate the Degree of Contamination from EO. Manuscript submitted for publication Retrieved from: http://www ejwagenmakers com/submitted/GronauMixtureModel pdf. 2015;.

9. Begg $\mathrm{CB}$, Mazumdar M. Operating characteristics of a rank correlation test for publication bias. Biometrics. 1994;50(4):1088-101. 
10. Egger M, Davey Smith G, Schneider M, Minder C. Bias in meta-analysis detected by a simple, graphical test. Bmj. 1997;315(7109):629-34. doi:10.1136/bmj.315.7109.629.

11. Friese M, Frankenbach J. p-Hacking and publication bias interact to distort meta-analytic effect size estimates. Psychological Methods. 2020;25(4):456.

12. Ioannidis JP, Trikalinos TA. An exploratory test for an excess of significant findings. Clin Trials. 2007;4(3):245-53. doi:10.1177/1740774507079441.

13. Simonsohn U, Nelson LD, Simmons JP. p-Curve and Effect Size: Correcting for Publication Bias Using Only Significant Results. Perspectives on Psychological Science. 2014;9(6):666-681. doi:10.1177/1745691614553988.

14. Benjamin DJ, Berger JO, Johannesson M, Nosek BA, Wagenmakers EJ, Berk R, et al. Redefine statistical significance. Nature Human Behaviour. 2018;2(1):6-10. doi:10.1038/s41562-017-0189-z.

15. Amrhein V, Greenland S. Remove, rather than redefine, statistical significance. Nature Human Behaviour. 2018;2(1):4-4. doi:10.1038/s41562-017-0224-0.

16. Morey RD, Romeijn JW, Rouder JN. The philosophy of Bayes factors and the quantification of statistical evidence. Journal of Mathematical Psychology. 2016;72:6-18. doi:https://doi.org/10.1016/j.jmp.2015.11.001.

17. Kass RE, Raftery AE. Bayes Factors. Journal of the American Statistical Association. 1995;90(430):773-795. doi:10.1080/01621459.1995.10476572.

18. Wagenmakers EJ. A practical solution to the pervasive problems ofp values. Psychonomic Bulletin and Review. 2007;14(5):779-804. doi:10.3758/BF03194105.

19. de Groot AD. The meaning of "significance" for different types of research [translated and annotated by Eric-Jan Wagenmakers, Denny Borsboom, Josine Verhagen, Rogier Kievit, Marjan Bakker, Angelique Cramer, Dora Matzke, Don Mellenbergh, and Han L. J. van der Maas]. Acta Psychologica. 2014;148:188-194. doi:https://doi.org/10.1016/j.actpsy.2014.02.001.

20. Chambers CD. Registered reports: a new publishing initiative at Cortex. Cortex. 2013;49(3):609-610. 
21. Steegen S, Tuerlinckx F, Gelman A, Vanpaemel W. Increasing Transparency Through a Multiverse Analysis. Perspectives on Psychological Science. 2016;11(5):702-712. doi:10.1177/1745691616658637.

22. Klein RA, Ratliff KA, Vianello M, Adams Jr RB, Bahník Š, Bernstein MJ, et al. Investigating variation in replicability: A "many labs" replication project. Social Psychology. 2014;45(3):142-152. doi:10.1027/1864-9335/a000178.

23. Robert CP, Cornuet JM, Marin JM, Pillai NS. Lack of confidence in approximate Bayesian computation model choice. Proceedings of the National Academy of Sciences of the United States of America. 2011;108(37):15112-15117. doi:10.1073/pnas.1102900108.

24. Szollosi A, Kellen D, Navarro DJ, Shiffrin R, van Rooij I, Van Zandt T, et al. Is Preregistration Worthwhile? Trends in Cognitive Sciences. 2020;24(2):94-95. doi:10.1016/j.tics.2019.11.009.

25. Fidler F, Wilcox J. -. In: Zalta EN, editor. Reproducibility of Scientific Results. Stanford Encyclopedia of Philosophy; 2018. p. -. Available from: https:

//plato.stanford.edu/archives/win2018/entries/scientific-reproducibility/

26. Ioannidis JPA. Why Most Published Research Findings Are False. PLOS Medicine. 2005;2(8):e124. doi:10.1371/journal.pmed.0020124.

27. Masicampo EJ, Lalande DR. A peculiar prevalence of $p$ values just below .05. Quarterly Journal of Experimental Psychology. 2012;65(11):2271-2279. doi:10.1080/17470218.2012.711335.

28. Hardwicke TE, Thibault RT, Kosie JE, Wallach JD, Kidwell MC, Ioannidis JPA. Estimating the Prevalence of Transparency and Reproducibility-Related Research Practices in Psychology (2014-2017). Perspectives on Psychological Science. 2021;0(0):1745691620979806. doi:10.1177/1745691620979806.

29. John LK, Loewenstein G, Prelec D. Measuring the Prevalence of Questionable Research Practices With Incentives for Truth Telling. Psychological Science. 2012;23(5):524-532. doi:10.1177/0956797611430953. 
30. Nosek BA, Ebersole CR, DeHaven AC, Mellor DT. The preregistration revolution. Proceedings of the National Academy of Sciences. 2018;115(11):2600. doi:10.1073/pnas.1708274114.

31. Rubin M. Does preregistration improve the credibility of research findings? The Quantitative Methods for Psychology. 2020;16(4):376-390. doi:10.20982/tqmp.16.4.p376.

32. Bakker M, van Dijk A, Wicherts JM. The Rules of the Game Called Psychological Science. Perspect Psychol Sci. 2012;7(6):543-54. doi:10.1177/1745691612459060.

33. Samejima F. 5. In: van der Linden WJ, Hambleton RK, editors. Graded Response Model. New York, NY: Springer New York; 1997. p. 85-100. Available from: https://doi.org/10.1007/978-1-4757-2691-6_5.

34. Embretson S, Reise S. Item Response Theory For Psychologists. vol. 4. Psychology Press; 2000.

35. Benjamin DJ, Berger JO, Johannesson M, Nosek BA, Wagenmakers EJ, Berk R, et al. Redefine statistical significance. Nature human behaviour. 2018;2(1):6-10.

36. Bakker M, Wicherts JM. Outlier removal, sum scores, and the inflation of the type I error rate in independent samples t tests: The power of alternatives and recommendations. Psychological methods. 2014;19(3):409.

37. Bar-Yam Y. General features of complex systems. Encyclopedia of Life Support Systems (EOLSS), UNESCO, EOLSS Publishers, Oxford, UK. 2002;1. 